\title{
The HCN molecule as a tracer of the nucleus rotation of comet 73P-C/Schwassmann-Wachmann $3^{\star}$
}

\author{
M. Drahus ${ }^{1}$, M. Küppers ${ }^{1,2}$, C. Jarchow ${ }^{1}$, L. Paganini ${ }^{1}$, P. Hartogh ${ }^{1}$, and G. L. Villanueva ${ }^{3}$ \\ 1 Max-Planck-Institut für Sonnensystemforschung, 37191 Katlenburg-Lindau, Germany \\ e-mail: drahus@mps.mpg.de \\ 2 European Space Astronomy Centre, ESA, 28691 Villanueva de la Cañada, Madrid, Spain \\ 3 NASA Goddard Space Flight Center, MD-20771 Greenbelt, USA
}

Received 19 October 2007 / Accepted 30 March 2009

\begin{abstract}
Context. The causes of cometary break-ups are still uncertain. One suggested mechanism is splitting due to fast rotation of the nucleus. This can be tested by measuring rotation periods of cometary fragments.

Aims. The exceptionally close approach of the split comet 73P/Schwassmann-Wachmann 3 to the Earth in May 2006 made it an ideal target to investigate the rotation of its fragments. We used the HCN light curve for this purpose, because it is particularly sensitive to the rotation of the nucleus and at the same time it allows us to study the physics of cometary activity.

Methods. Comet 73P/Schwassmann-Wachmann 3 was observed between May 1 and 22, 2006, with the Submillimeter Telescope on Mt. Graham, Arizona, USA. Emission from HCN and CS were clearly detected. In this work we focus exclusively on the observations of the HCN molecule in fragment C, obtained during five nights between May 10 and 22, 2006, which provide the best $S / N$ and the best temporal coverage.

Results. The light curve of comet 73P-C/Schwassmann-Wachmann 3 in HCN shows strong non-random variations, most probably stimulated by the nucleus rotation. The variability has an amplitude of about a factor of 2 on a time scale of hours. Among several plausible solutions for periodicity, we found strong indications for a rotation period of between 3.0 and $3.4 \mathrm{~h}$, consistent with the determination from the Hubble Space Telescope. At 1 AU from the Sun the mean-diurnal HCN production rate was $2.7 \times 10^{25} \mathrm{molec} \mathrm{s}^{-1}$ (with an uncertainty of about 20\%) and the coma was expanding with a velocity of $0.8 \pm 0.1 \mathrm{~km} \mathrm{~s}^{-1}$. The line position was evolving with a phase angle that is visible in the night-averaged spectra.

Conclusions. Evolution of the line position is consistent with the solar-stimulated activity. The mean-diurnal HCN production rate should be considered as very high, and it requires an unusually large fraction of the nucleus area to be active, whereas the coma expansion velocity was typical. The proposed rotation period, being the shortest ever determined for a cometary nucleus, cautiously suggests the disruption of the parent body due to a large centrifugal force, though it cannot be considered as a proof of this scenario. On the other hand, the observed stability of 73P-C against the rotational disruption suggests a bulk tensile strength of at least 14-45 Pa. The rotation period was surprisingly stable, indicating that no more than about $0.2 \%$ of the total outgassing was effectively accelerating or decelerating the nucleus spin. This is consistent with the large active fraction of the nucleus.
\end{abstract}

Key words. comets: individual: 73P-C/Schwassmann-Wachmann 3 - submillimeter: planetary systems

\section{Introduction}

More than 40 comets are known to have split, producing several hundred fragments (Boehnhardt 2004). However, except for rare cases of cometary disruption by tidal forces due to close approaches to the Sun or to Jupiter, the causes of cometary splitting are still unknown. One candidate mechanism is rotational breakup, when the centrifugal force surpasses the nucleus self gravity and tensile strength (cf. e.g. Davidsson 1999, 2001).

The hypothesis of rotational splitting is testable: a rotation period of a main fragment, which is close to the disruption threshold shortly after the break-up, would be suggestive of rotational splitting. On the other hand, a long rotation period after break-up does not necessarily exclude rotational splitting, because cometary nuclei may change their spin state very rapidly, especially those highly active and of a small size (cf. e.g. Samarasinha et al. 1986; Jewitt 1999; Gutiérrez et al. 2002).

* Appendices are only available in electronic form at http://www . aanda. org
Comet 73P/Schwassmann-Wachmann 3 (hereafter SW3) was discovered in 1930 by Arnold Schwassmann and Arno Arthur Wachmann (Hamburg Observatory, Germany), around the perigee of its orbit at only $0.06 \mathrm{AU}$ from the Earth. It is a short-period Jupiter Family Comet with an orbital period of 5.36 years and a perihelion distance of $0.94 \mathrm{AU}$. The comet broke up into at least four pieces in 1995 (Boehnhardt \& Kaufl 1995; Scotti et al. 1996), and the splitting was accompanied by an outburst (Crovisier et al. 1995; Hale et al. 1995; Morris et al. 1995). Three of the components were re-detected during their next perihelion passage in 2000/2001 (Kadota et al. 2000). While the geometry of the apparition of SW3 in 2000/2001 was unfavorable, the comet approached the Earth to less than $0.1 \mathrm{AU}$ in May 2006, displaying over 60 fragments (Weaver 2006). Of all of them, fragment $\mathrm{C}$ is the largest, about $0.5 \mathrm{~km}$ in radius (Toth et al. 2005, 2006; Nolan et al. 2006).

The exceptionally close approach of comet SW3 was a good opportunity to search for variability in the emission of parent molecules, stimulated by the nucleus rotation. Using a typical 
(sub-) millimeter facility, the telescope beam radius was of the order of $1000 \mathrm{~km}$ at the comet, ensuring that most of the observed emission originated from the inner coma, so that most of the material left the nucleus within less than an hour before the observation. Therefore periodicities with a period longer than one hour might have been detected.

We undertook such study using the $\mathrm{HCN}$ molecule, because it is believed to sublimate directly from the nucleus, and it offers the brightest emission lines for ground-based (sub-) millimeter observations. This makes it ideal for investigating rotation of a cometary nucleus. According to Huebner et al. (1992), 97\% of the molecules photodissociate into $\mathrm{H}$ and $\mathrm{CN}$, with a lifetime of $22 \mathrm{~h}$ at $1 \mathrm{AU}$ from the (quiet) Sun. This corresponds to a scale-length of $60000 \mathrm{~km}$, assuming a typical gas expansion velocity at this heliocentric distance. Furthermore, $\mathrm{CN}$ is one of the brightest radicals observed in cometary spectra in the visible range, thus the $\mathrm{HCN}-\mathrm{CN}$ connection is the best studied parent-daughter relation in cometary comae. However, it is still not clear if $\mathrm{HCN}$ is the only important parent of $\mathrm{CN}$ (see e.g. Bockelée-Morvan \& Crovisier 1985; Fray et al. 2005). The production rate of $\mathrm{HCN}$ is of the order of $0.1 \%$ relative to water, and this ratio is remarkably constant over large range of heliocentric distances and very similar for many comets (cf. Biver et al. 2002b; Bockelée-Morvan et al. 2004a).

In this article we show selected results from our observations of comet SW3 at (sub-) millimeter wavelengths in May 2006. In Sect. 2 we present our data and outline the reduction steps. Section 3 shows an evolution of the line position with phase angle. In Sect. 4 we determine and discuss the production rates of $\mathrm{HCN}$, and in Sect. 5 we use them to investigate the rotation of the nucleus. Finally, Sect. 6 summarizes this work, and presents our conclusions for future studies. Sections 4 and 5 are supplemented with Appendices A-C, which contain a detailed justification of our work. Appendix D contains the complete data set which we used for this study.

\section{Observations and data reduction}

We monitored comet SW3 at (sub-) millimeter wavelengths between May 1 and 22, 2006, searching for different molecules in the two brightest fragments (designated as C and B). However, in this article we focus only on the results of our observations of the $\mathrm{HCN}$ emission from fragment $\mathrm{C}$ (hereafter SW3-C).

We used the 10.0-m Heinrich-Hertz Submillimeter Telescope (Baars \& Martin 1996; Baars et al. 1999) at Mt. Graham International Observatory in Arizona (hereafter SMT). Depending on atmospheric transmission we either used the $1.3-\mathrm{mm}$ JT single-sideband SIS receiver or the $345-\mathrm{GHz}$ double-sideband SIS receiver, to observe $\mathrm{HCN}$ through the $J(3-2)$ or $J(4-3)$ rotational transitions. Both receivers are socalled dual channel receivers, allowing us to observe the source object in horizontal and vertical polarization simultaneously.

Spectral analysis was performed using the facility AcoustoOptical Spectrometers AOS-A and AOS-B with a total bandwidth of $1 \mathrm{GHz}$ and 2048 nominal channels each; however, the real resolution of the spectrometers is $934 \mathrm{kHz}$ for AOS-A and $913 \mathrm{kHz}$ for AOS-B, so the linear CCD elements oversample the spectra by about a factor of 2 . These two spectrometers were always connected to the two channels of the receivers, allowing us to improve the $S / N$ by averaging intermediate results from the horizontal and vertical polarizations. Co-adding the original spectra from different polarizations was problematic due to the different arrangement and slightly different width of the spectral channels in each spectrometer.

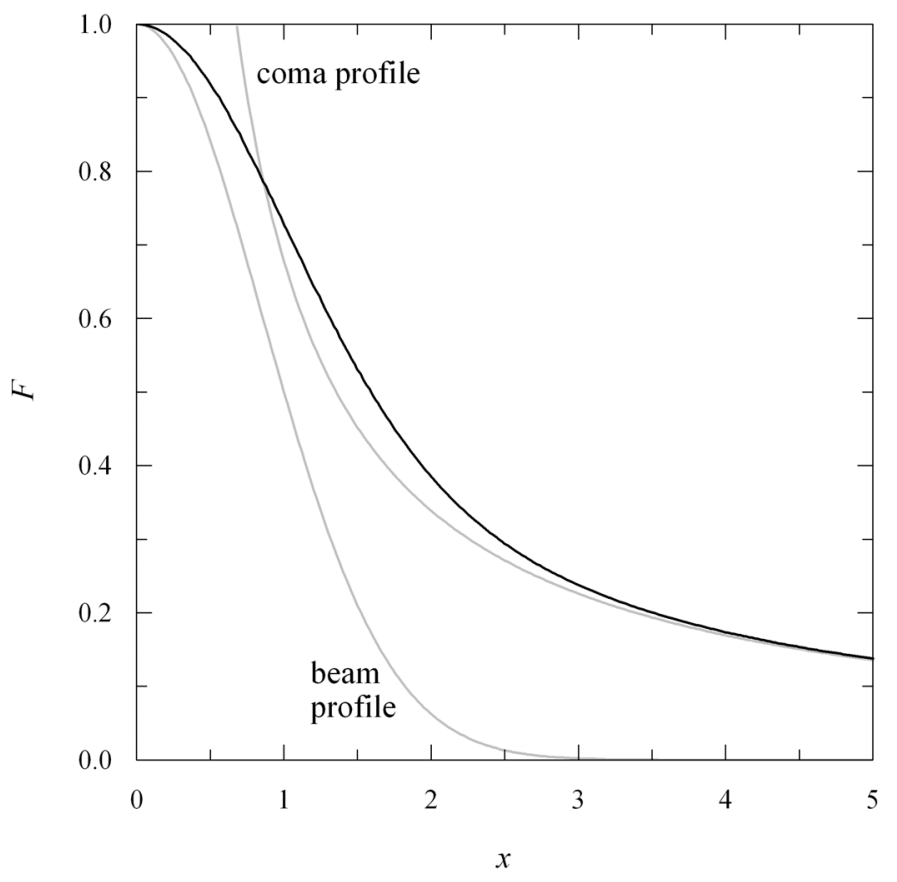

Fig. 1. Loss of the normalized flux $F$ due to a relative pointing offset $x$ (measured in units of the beam radius at half-power). The dependence was derived for our model coma (further described in Sect. 4.1) and for the assumed beam pattern (see Eq. (1)), both profiles being shown for comparison.

For all derivations we approximate the beam as a symmetric Gaussian, whose $F W H M$ is given (in radians) by:

$F W H M=\frac{c b}{v D}$

where $c$ is the speed of light, $v$ is observed frequency, $D$ is dish diameter, equal to $10 \mathrm{~m}$ for the SMT, and $b$ is a dimensionless factor, intrinsic to every dish, which is equal to 1.25 for the SMT. The half-power angular radius of the beam was therefore 14.5" at the lower frequency and $11^{\prime \prime}$ at the higher one.

The position of the comet was continually calculated from the orbital elements provided by the JPL HORIZONS system ${ }^{1}$ for the epoch of our observations. The pointing was calibrated on Jupiter, Saturn, and most frequently on the protoplanetary nebula CRL2688 that was close to the comet. From the stability of these observations we concluded that the pointing error was at worst of the order of half the beam radius at half-power ( 7 " or $5^{\prime \prime}$ depending on the observed frequency) for the observations accepted for analysis (see further in this section). Such precision is an order of magnitude worse than that guaranteed by the ephemeris but it still ensured that the flux loss was below 10\% (see Fig. 1).

The spectra of the comet were taken in a position-switching mode, using a $0.5^{\circ}$ offset in azimuth where the coma contamination is negligible. We used a $15 \mathrm{sec}$ integration time for the $\mathrm{ON}$ and also a $15 \mathrm{~s}$ integration time for the OFF position, and each spectrum was composed of 8 or $10 \mathrm{ON}-\mathrm{OFF}$ pairs, thus giving us a fundamental time resolution of 4 or 5 min (hereafter exposure).

The moments of observations were not corrected for the travel time of light as the correction was negligible (see the geocentric distances in Table 1). They are approximately mid-points of the exposures, but not exactly: we added halves of the exposures to the initialization moments of the spectra, but neglected

1 http://ssd.jpl.nasa.gov/?horizons 
Table 1. Journal of the observations.

\begin{tabular}{ccccccccrr}
\hline \hline $\begin{array}{c}\text { Date UT } \\
\text { (May 2006) }\end{array}$ & Target line & $\mathcal{N}^{b}$ & $\begin{array}{c}\text { Total exposure }^{c} \\
{[\mathrm{~min}]}\end{array}$ & $\begin{array}{c}r^{d} \\
{[\mathrm{AU}]}\end{array}$ & \multicolumn{1}{c}{$\begin{array}{c}\Delta^{e} \\
{[\mathrm{AU}]}\end{array}$} & \multicolumn{1}{c}{$\begin{array}{c}\phi^{f} \\
{\left[{ }^{\circ}\right]}\end{array}$} & $\begin{array}{c}\lambda^{g} \\
{\left[{ }^{\circ}\right]}\end{array}$ & $\begin{array}{r}\beta^{h} \\
{\left[{ }^{\circ}\right]}\end{array}$ & $\begin{array}{r}\text { Beam }^{i} \\
{[\mathrm{~km}]}\end{array}$ \\
\hline 10.4979 & $J(4-3)$ & 44 & 176 & 1.0214 & 0.0803 & 79.445 & 310.247 & 49.912 & 635 \\
11.6885 & $J(3-2)$ & 13 & 65 & 1.0148 & 0.0789 & 84.287 & 319.050 & 45.545 & 831 \\
12.5524 & $J(3-2)$ & 15 & 75 & 1.0101 & 0.0786 & 87.816 & 324.738 & 41.924 & 829 \\
20.6222 & $J(3-2)$ & 41 & 205 & 0.9732 & 0.1054 & 108.715 & 354.173 & 9.206 & 1112 \\
22.4882 & $J(3-2)$ & 11 & 55 & 0.9664 & 0.1167 & 110.039 & 357.585 & 4.196 & 1231 \\
\hline
\end{tabular}

${ }^{a}$ Middle moment of an individual observing run; ${ }^{b}$ number of observations; ${ }^{c}$ individual exposures were consequently 4 min (first night) and 5 min (other nights); ${ }^{d}$ heliocentric distance; ${ }^{e}$ geocentric distance; ${ }^{f}$ phase angle; ${ }^{g}$ ecliptic longitude; ${ }^{h}$ ecliptic latitude; ${ }^{i}$ beam radius at half-power at comet's distance.

the time taken by the initial calibrations, switching time, time losses when the telescope was blown off the position, etc.

The absolute temperature scale was calibrated using the chopper wheel method (see e.g. Ulich \& Haas 1976; Kutner \& Ulich 1981), which automatically corrects for absorption in the Earth's atmosphere, resistive antenna losses, and rearward spillover and scattering terminated at ambient temperature. Furthermore, we corrected the temperature scale for the main beam efficiency, which we found to be equal to $0.60 \pm 0.04$ (at both monitored frequencies) using our observations of continuum in Venus, Mars, Jupiter, and Saturn.

Because a typical edge tapering of $12 \mathrm{~dB}$ reduces the maximum power level of the first sidelobe to less than $0.3 \%$ of the power level of the main beam, the sidelobes do not contribute significantly to the convolution result of the the antenna pattern with planetary discs or coma brightness distribution. Furthermore, as long as the main beam pattern is similar to the assumed Gaussian profile (which is the case for e.g. an edgetapered Airy profile), the influence of a small error in the assumed beam width on cometary observations will be largely compensated for by the main beam efficiency factor (affected similarly if calculated using the same assumed beam).

The frequency scale was converted to the radial-velocity scale (negative velocities indicate blueshift), and corrected for the radial velocity of the comet with respect to the telescope. We used $265.88643392 \mathrm{GHz}$ as the central rest frequency of the $J(3-2)$ transition, and $354.50548084 \mathrm{GHz}$ for the $J(4-3)$ one. These frequencies are the optocenters of the complete hyperfine structures as listed in the Cologne Database for Molecular Spectroscopy $^{2}$ (Müller et al. 2005).

For each single spectrum the line area $\int T_{\mathrm{mB}} \mathrm{d} v$ was determined from a velocity interval between -3 and $+3 \mathrm{~km} \mathrm{~s}^{-1}$, and two symmetric intervals from -10 to $-3 \mathrm{~km} \mathrm{~s}^{-1}$ and from +3 to $+10 \mathrm{~km} \mathrm{~s}^{-1}$ were used to estimate the background level. The background was subtracted upon fitting a linear baseline with a $3 \sigma$ iterative rejection of "bad" spectral channels. Additionally, information about noise in the background was used to estimate a statistical error of the line area, assuming that both the line and the background have identical noise. After this procedure was applied separately to the spectra from AOS-A and AOS-B, the resulting line areas were averaged (with the same statistical

\footnotetext{
2 Available online at http://www.astro.uni-koeln.de/site/ vorhersagen/. Note that the main database does not resolve the hyperfine components of the HCN lines. The resolved hyperfine structure of the first five rotational transitions is available separately, at http://www.astro.uni-koeln.de/cdms/daten/HCN/ $\mathrm{HCN} / \mathrm{CQ27501}$ hfs . cat. The frequency of the $J(4-3)$ transition in the main database corresponds to one of the hyperfine components, and it slightly differs from the optocenter of the whole structure.
}

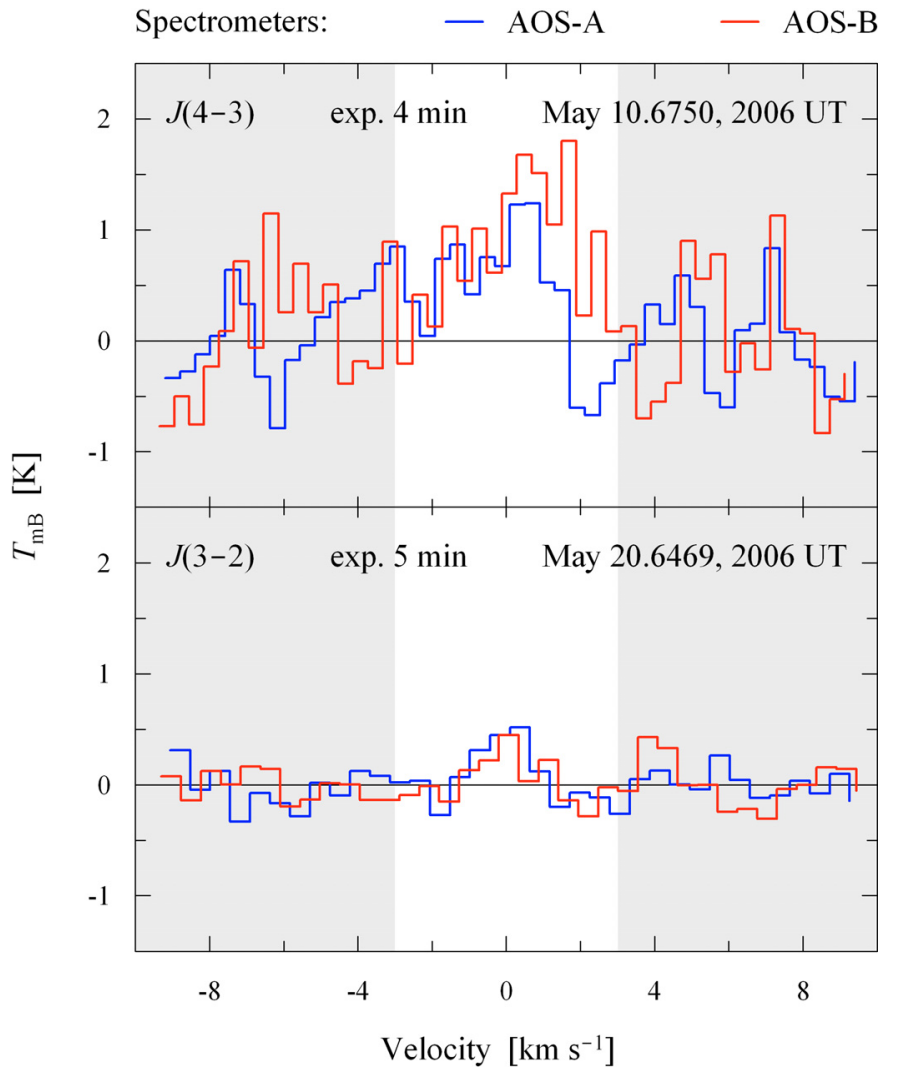

Fig. 2. Examples of individual calibrated spectra. The panels show the spectra with maximum (upper) and minimum (lower) mean value of $\int T_{\mathrm{mB}} \mathrm{d} v$ from both AOS spectrometers. The white region shows the range used for calculating the line area, and the gray regions show the ranges used for fitting the background.

weights), and the effective errors were calculated. Figure 2 shows examples of such individual spectra for both the $J(3-2)$ and the $J(4-3)$ transitions. Since the effect of oversampling is not clearly visible, possibly due to an additional source of noise which partly removed the correlation between neighboring channels, for simplicity we analyzed the spectra as their channels were completely independent.

To ensure consistency of the absolute calibration and stability of the instrumentation, we periodically (about each $90 \mathrm{~min}$ ) monitored standard calibration sources on the sky, namely CRL2688, W51D, DR21, and W3(OH). Within one night and for a fixed instrumental setup we found intensity drops of up to $50 \%$, which we believe to be caused directly or indirectly (via 


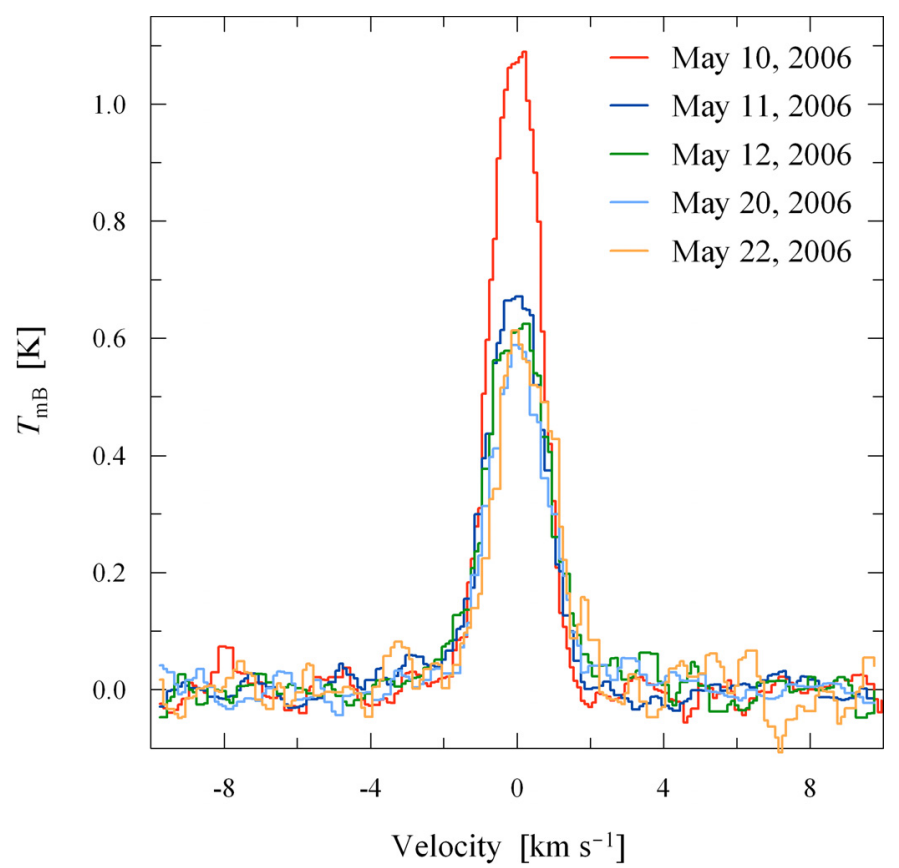

Fig. 3. Night-averaged calibrated spectra. The line for May 10, 2006 (red line) is more intense than the others because it results from a different transition (cf. discussion in Appendix A.6). The total exposure for each night is listed in Table 1 . The constituent spectra were re-binned to a common arrangement of channels, each $0.1 \mathrm{~km} \mathrm{~s}^{-1}$ wide, which made it possible to average the spectra from AOS-A and AOS-B. Note that these new channels are not independent.

pointing calibration) by anomalous refraction (Altenhoff et al. 1987). To ensure a consistent and properly calibrated time series of cometary HCN intensity, we restricted our observations in the following way:

(i) we rejected the nights suffering from an insufficient monitoring of the calibration sources;

(ii) we rejected the nights during which we encountered instabilities of the calibration sources larger than $20 \%$;

(iii) post-factum we restricted the time series to the measurements with the production rate errors below $1.2 \times$ $10^{25}$ molec s$^{-1}$ (calculation of the production rates is further described in Sect. 4.1) and hence rejected 7 observations; additionally, we rejected the last 3 observations from May 10, 2006, whose errors are significantly higher than those of the earlier data from that date.

Finally, we obtained 124 individual measurements of the line area $\int T_{\mathrm{mB}} \mathrm{d} v$ (Table D. 1 in Appendix D), collected over 5 days (Table 1), which we used for further analysis. The errors on the line area include only the statistical component, since the other error sources, discussed in this section, are of a different nature (systematic and/or maximum) and are often negligibly small. Visual inspection of Table D.1 shows that occasionally a difference between neighboring measurements exceeds very significantly an acceptable range indicated by the errors. Although we cannot explain this effect, we assume it has a negligible influence on our analysis due to its rareness and apparently stochastic nature. Additionally, in Fig. 3 we present the weighted-mean spectra, obtained upon averaging the selected observations separately for each night, where the weights were inversely proportional to the square of the background noise.
Date 2006 (0 UT)

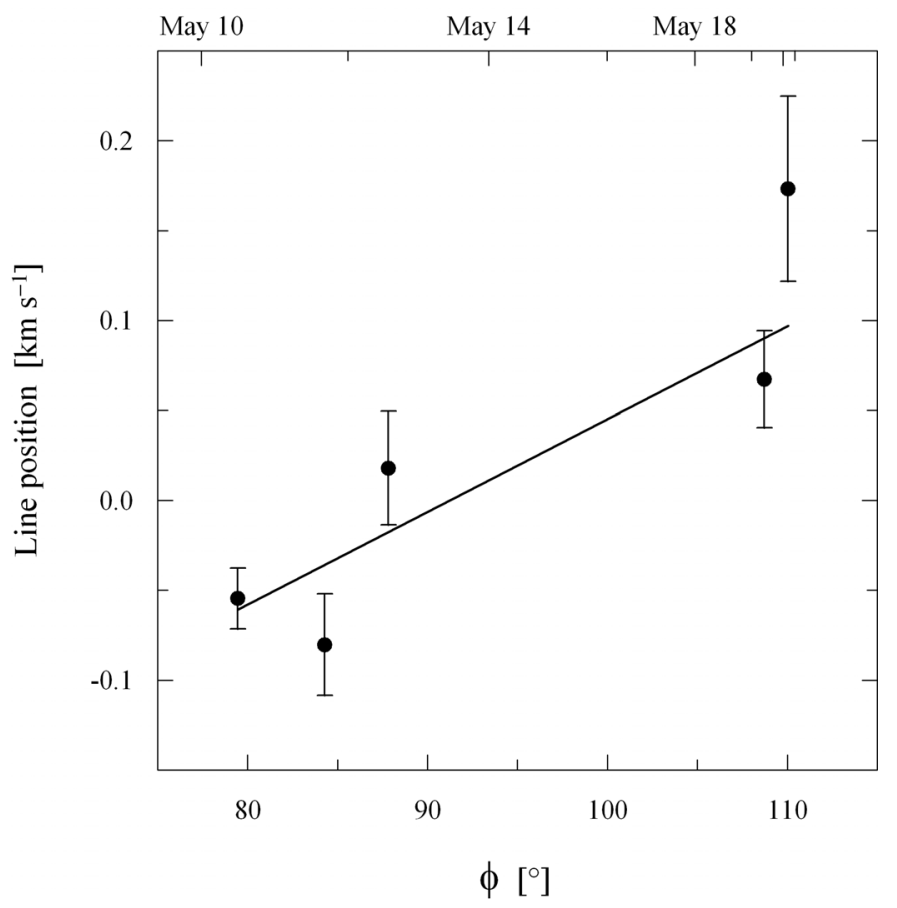

Fig. 4. Evolution of the line position in the night-averaged spectra (illustrated in Fig. 3) with phase angle $\phi$. The solid line shows a weighted linear fit to guide the eye.

\section{Line position in the night-averaged spectra}

Using the night-averaged spectra (illustrated in Fig. 3) we calculated positions of the lines (on a common velocity scale), defined as points splitting line area into halves. An error of the position was estimated using the Monte Carlo approach: on each spectrum we superimposed 1000 noise realizations, and in each individual simulation we determined the line position. The scatter of the obtained values is a very good measure of the investigated uncertainty, therefore, as the error we adopted the standard deviation calculated with respect to the original line position. While simulating noise we assumed a Gaussian noise distribution, with a variance determined from the background noise (see Sect. 2) thus identical for all the channels within one spectrum. Although as the references for the simulations we ideally should have used noiseless representations of the real data, in this specific case Gaussian fits featured noticeably shifted centers, thus we used the investigated spectra themselves. We followed these procedures separately for the night-averaged spectra from AOS-A and AOS-B, hence preserving the original arrangement of their channels. Finally we determined the mean positions from both spectrometers, which are presented versus phase angle $\phi$ in Fig. 4.

It is clearly visible that the lines became redder with the increasing phase angle $\phi$. This effect is expected for outgassing stimulated by the Sun, whose position controls the temperature, and hence the sublimation rate, over the nucleus. Moreover, the line position equal to nearly zero at $\phi=90^{\circ}$ indicates that at that moment the distribution of the outgassing rate was symmetric with respect to some direction perpendicular to the line of sight (reaching a maximum in this direction), which might have been exactly Sunward. The scatter of the line positions about the global trend well corresponds to their errors estimated from the background noise (which would be even slightly underestimated 
if the spectral channels were partly correlated - see Sect. 2). Clearly, the observed trend contains a lot more information about the activity of this comet, however, in order to retrieve them we would have to model the complete line profiles in the individual (i.e. not night-averaged) spectra, which is impossible due to the too large noise. Note that this effect should be accompanied by an evolution of the line area $\int T_{\mathrm{mB}} \mathrm{d} v$ with phase angle $\phi$, as we model it in Appendix A.4, but we did not investigate our data in this respect due to its small magnitude and because it partly overlaps with the heliocentric trend (see Sect. 4.2).

\section{Production rate of the $\mathrm{HCN}$ molecule}

\subsection{Model and parameters}

In order to retrieve a production rate $Q$ (that is the global outgassing rate of a given molecule) from a measurement of the line area $\int T_{\mathrm{mB}} \mathrm{d} v$, a model involving kinematics, chemistry, and excitation of the molecules, along with light propagation properties, must be used. For the purpose of our derivation we assume that:

(i) the coma is optically thin at the observed frequencies;

(ii) LTE conditions are present throughout the coma;

(iii) the volume density of the molecules is inversely proportional to the square of the nucleocentric distance, i.e. the photodissociation process is neglected;

(iv) the molecules are isotropically ejected from the nucleus;

(v) the production rate $Q$ is constant in time;

(vi) the expansion velocity $v_{\exp }$ and the temperature $T$ of the molecules are constant in space and time; under LTE, the excitation temperature is equal to the kinetic temperature, therefore we simply refer to the gas temperature.

Under these assumptions the production rate $Q$ depends linearly on the line area $\int T_{\mathrm{mB}} \mathrm{d} v$ :

$Q=\frac{2}{\sqrt{\pi \ln 2}} \frac{k}{h} \frac{b \Delta v_{\exp }}{D I(T) v}\left(\mathrm{e}^{h v / k T}-1\right) \int T_{\mathrm{mB}} \mathrm{d} v$,

where $k$ and $h$ are the Boltzmann and the Planck constants respectively, $\Delta$ is geocentric distance, and $I(T)$ is the integrated line intensity (at temperature $T$ ) as defined in the JPL spectral line catalog 3 (Pickett et al. 1998). In this equation the same approximation of the beam is used as given by Eq. (1). Note that in order to calculate $Q$ in molecules per second, $\int T_{\mathrm{mB}} \mathrm{d} v$ must be provided in the commonly used unit of $\mathrm{K} \mathrm{m} \mathrm{s}^{-1}$.

Unfortunately, we could not observe several HCN lines simultaneously. This would have enabled us to derive the gas temperature $T$ by solving Eq. (2) simultaneously for the production rate and the temperature itself. Instead, we adopted $T=80 \mathrm{~K}$.

Supplying our model with this temperature we calculated the HCN expansion velocity $v_{\text {exp }}$ using the line widths determined from the night-averaged spectra (Fig. 3). We found $v_{\exp }=0.8 \pm$ $0.1 \mathrm{~km} \mathrm{~s}^{-1}$, which is a mean value from the results obtained individually for each single night, and its error is the standard deviation among the constituent measurements.

A detailed justification of the assumptions of our model and of the input parameters is presented in Appendix A.

\footnotetext{
${ }^{3}$ Available online at http://spec.jpl.nasa.gov/. Note that the integrated line intensity is provided for $T=300 \mathrm{~K}$, hence requires an adequate scaling to a lower temperature if a realistic cometary gas is considered.
}

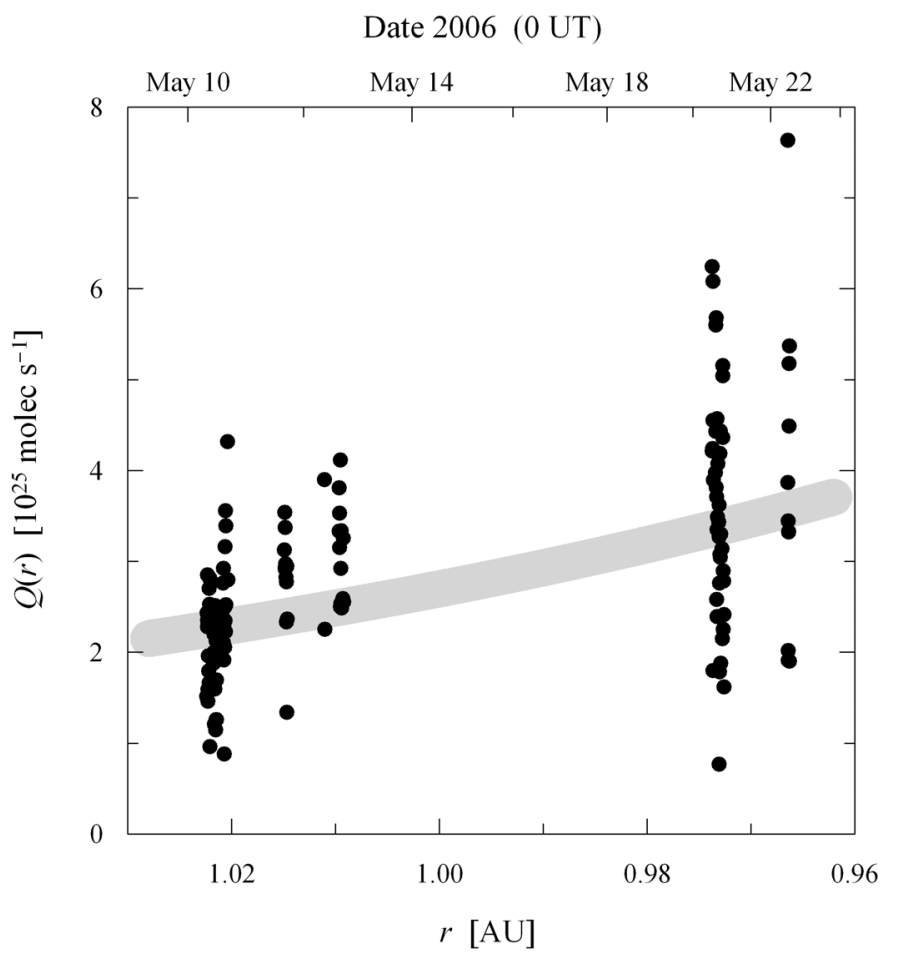

Fig. 5. Evolution of the HCN production rate $Q$ with heliocentric distance $r$. The gray line shows the best power-law fit.

\subsection{Individual production rates}

Using the simple model (Sect. 4.1) we converted each individual measurement of the line area $\int T_{\mathrm{mB}} \mathrm{d} v$ (see Table D.1) into the corresponding $\mathrm{HCN}$ production rate $Q$, and analyzed how they change with the heliocentric distance $r$ of the comet (see Fig. 5). An unweighted power-law fit to this dependence yields the power index of $-8.2 \pm 1.4$ and the mean production rate $\left\langle Q_{0}\right\rangle=(2.70 \pm 0.09) \times 10^{25}$ molec s$^{-1}$ at $r=1 \mathrm{AU}$ (indicated by index "zero"), corresponding to $1.22 \pm 0.04 \mathrm{~kg} \mathrm{~s}^{-1}$. Since heliocentric evolution of the HCN production rate typically follows power laws with indices between 2 and 5 (see e.g. Biver et al. 1999, 2002a), the obtained dependence should be considered as unusually steep. The likely reason for this difference is the very limited range of heliocentric distances covered by our observations, thus the obtained evolution shows only an instantaneous behavior of this comet.

Nevertheless, the ultimate goal of obtaining this dependence was to remove the instantaneous heliocentric trend from the production rate in order to search for its cyclic changes that might have been driven by the nucleus rotation. As further shown in Sect. 5, this effect may be responsible for the large scatter of the measurements about the fit. Thus, the presented snapshot of the heliocentric dependence concerns, strictly speaking, the mean diurnal production rate, provided that the rotation phase was sampled fairly uniformly. This is discussed in more detail in Appendix B.

This also means that the derived errors of the fit parameters resulting purely from the scatter of the measurements - are artificially boosted by the short-term variability of the production rate about its mean-diurnal value. On the other hand, they do not account (at least not directly) for the simplifications of our model and the uncertainties of its input parameters (Sect. 4.1), the instrumental effects and the individual errors resulting from 
the noise statistics (Sect. 2), and sampling of the nucleus rotation phases (Appendix B).

Using the determined trend we reduced all the individual measurements to $r=1 \mathrm{AU}$, and denoted them as $Q_{0}$. Their errors are the statistical uncertainties on the line area $\int T_{\mathrm{mB}} \mathrm{d} v$ (see Sect. 2) converted with Eq. (2) and reduced with the same trend. Hence they neither account for the several effects discussed in Sect. 2, nor for the simplifications of our model (Sect. 4.1) or the uncertainty on the heliocentric trend derived in this section. The reason is that all these effects are of a different nature (systematic and/or maximum), whereas it is the relative uncertainty between the individual $Q_{0}$, not the absolute one, which matters for the periodicity analysis.

\subsection{Discussion}

The determined expansion velocity is typical for a comet at this heliocentric distance (cf. e.g. Biver et al. 1999, 2002a), and is consistent with theoretical models (see e.g. Bockelée-Morvan \& Crovisier 1987).

The mean diurnal $\mathrm{HCN}$ production rate at $r=1 \mathrm{AU}$ is generally consistent with other corresponding determinations, published by Milam et al. (2006), Villanueva et al. (2006), and Dello Russo et al. (2007), though discrepancies up to a factor of 3 exist. They can be explained by different heliocentric distances and observing geometries of the comet (the latter being further discussed in Appendix A.4), along with modulation induced by the nucleus rotation (further discussed in Sect. 5). Inconsistencies between the different techniques also cannot be excluded (cf. e.g. Magee-Sauer et al. 1999; Fray et al. 2005).

Furthermore, we compared our result with the corresponding values for eight other comets. Whenever necessary we normalized their production rates using a power-law heliocentric dependence with an exponent of -4 . This is between the values of -4.5 and -3.4 , obtained from the long-term pre- and post-perihelion evolution of the HCN production rate in comet Hale-Bopp (Biver et al. 2002a), though the slope was noticeably flatter for comet Hyakutake (Biver et al. 1999). The correction was significant only for comet Tempel 1 (observed at $r=1.62 \mathrm{AU}$ ), and moderate for comet Borrelly (observed at $r=1.36 \mathrm{AU}$ ). The comparison is presented in Fig. 6, where the production rates $Q_{0}$ are plotted vs. the nuclei radii $R$, along with the best fit of a square function (calculated in logarithmic space); that is because the total nucleus area is the main parameter controlling the global sublimation. The dependence shows that the HCN production rate in SW3-C was unusually high. Since the relative abundance of this molecule was found to be typical (Villanueva et al. 2006), only an exceptionally high nucleus volatility - that is a large active fraction - can explain this. The conclusion is on one hand not surprising, bearing in mind the former break-up which excavated much fresh and highly volatile material; on the other hand, the comet has been continually losing its excessive activity since then (Boehnhardt 2004), and even though, it was still unusually active a decade later. The dependence also shows that at $r=1$ AU a typical nucleus with the radius of $R=1 \mathrm{~km}$ produces $\mathrm{HCN}$ at the rate of $1.5_{-1.3}^{+8.7} \times 10^{25}$ molec s $^{-1}$ (corresponding to $0.7_{-0.6}^{+3.9} \mathrm{~kg} \mathrm{~s}^{-1}$ ). Covering nearly 4 orders of the nucleus area, the analysis is fairly insensitive to the errors on individual data points, introduced by observing noise, particular models, normalizations, etc. Therefore, the scatter of the points is most likely dominated by diversity among the nucleus volatilities, with some contamination from differences in composition and shape.

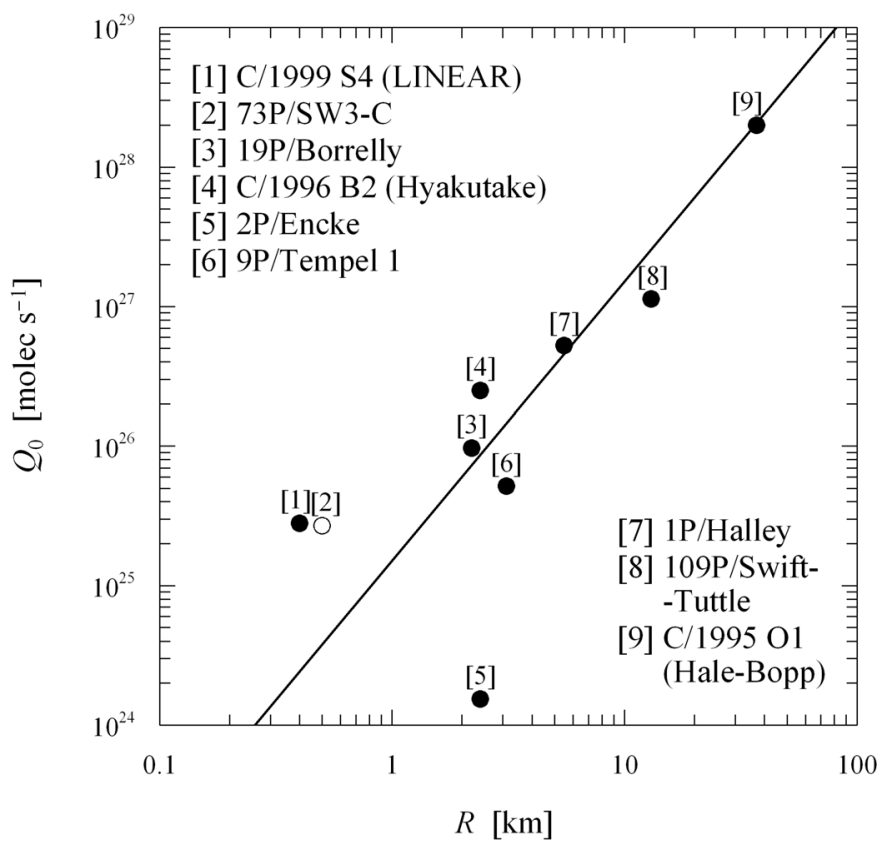

Fig. 6. Compilation of the $\mathrm{HCN}$ production rates $Q_{0}$ (normalized to $r=1 \mathrm{AU}$ ) for comets with known nucleus radius $R$. The solid line shows the fit of a square function. Except for SW3-C (see references in Sect. 1), the radii were taken from Lamy et al. (2004), and the production rates from: [1] Bockelée-Morvan et al. (2001); [2] this work (see Sect. 4.2); [3] Bockelée-Morvan et al. (2004b); [4] Biver et al. (1999); [5] Woodney et al. (2003); [6] Biver et al. (2007); [7] Schloerb et al. (1986); [8] Bockelée-Morvan et al. (1994); and [9] Biver et al. (2002a).

\section{Rotation period of the nucleus}

\subsection{Basic concepts}

An active cometary nucleus may manifest its rotation by stimulating a periodic variation of the global outgassing rate, induced by diurnal changes of the insolation over the active region(s). Also a uniformly volatile nucleus may produce such variability provided that its shape is irregular - hence the effective sublimation area varies as the insolation changes during the rotation cycle. In principle, for both scenarios the observed rotation phase profile of the production rate may be noticeably different from the one which is intrinsic to the nucleus; they should be, however, quite similar in our case (see Appendix A.5).

Rotation periods have been determined for many cometary nuclei, both active and inactive, using several different techniques (see e.g. Jorda \& Gutiérrez 2000; Samarasinha et al. 2004). However, periodic variability of the parent molecule production rate, stimulated by the nucleus rotation, has been detected only very recently, by Biver et al. (2007) for $\mathrm{HCN}$ in comet 9P/Tempel 1 (the Deep Impact target). Although the rotation period they found is consistent with the determination from optical photometry of the bare nucleus (Lamy et al. 2007) - thus providing a double-peak phasing of the light curve - the phase profile for $\mathrm{HCN}$ features only one peak. Moreover, this profile is well correlated with periodic oscillations of the line position, which were also detected by Biver et al. (2007). This demonstrates that the phase profiles for the $\mathrm{HCN}$ production rate and for the optical light curve are indeed controlled by different underlying mechanisms. 
A clear advantage of using a time series of parent molecule production rate to investigate the rotation of active comets is high probability of detecting a phase profile with a large amplitude $A$ (which we define as a ratio of maximum to minimum of the phase profile). This will be the case if the sublimation is indeed restricted to a limited number of isolated sources, and the observations closely reflect an intrinsic activity pattern of the nucleus, satisfying the conditions discussed in Appendix A.5. This contrasts with low amplitudes of the phase profiles for daughter species and for dust. The former one is damped by the significant excess velocities (in random directions) gained during their photodissociative creation, and also due to the exponential nature and long time scale of this process, both effects making the spacetime distribution of daughter species more uniform. The latter is damped because of low and simultaneously sizedependent velocities of dust grains; it was also postulated that dust ejection may be driven more by sublimation of the most volatile ices $\left(\mathrm{CO}, \mathrm{CO}_{2}\right)$ than by water ice (Feaga et al. 2007), which is weakly sensitive to the diurnal changes of the insolation conditions. Moreover, if a large field of view or long exposures are used, then the rotation-driven variability of any coma component (i.e., daughter species, dust, or parent molecules) is additionally damped (cf. Appendix A.5). An example of these effects are observations of comet Tempel 1: compare the HCN phase profile $(A=2.3)$ reported by Biver et al. (2007) - although it is expected to differ noticeably from the intrinsic one (thus they call it apparent $)$ - with the $\mathrm{CN}$ and dust phase profiles $(A=1.5$ and $A<1.5$ respectively) inferred by Manfroid et al. (2007).

Another approach is to investigate a (diurnal) variability of the outgassing direction instead of (or along with) variations of the production rates. Such an effect stimulates the variability of the line position and shape over the rotation cycle. Requiring spectral resolutions of at least one million (for realistic gas outflow velocities in comets), this approach is feasible only for radio techniques. The rotation-driven variability of the line shape and/or position was first identified for comet Hale-Bopp (Henry et al. 2002; Boissier et al. 2007), and later found for Tempel 1 (Biver et al. 2007).

Unfortunately, investigating periodicity of the line positions measured in our individual spectra of SW3-C we found only random noise. Although an evolution with phase angle is evident in the night-averaged spectra (see Sect. 3), there is no hint of the rotation-driven variability also there, though it might have manifested itself by producing an excess scatter in the trend (if different rotation phases of the nucleus were sampled on different nights). A possible explanation for this non-detection is simply an insufficient $S / N$ of the individual spectra, causing too large an uncertainty on the line position. Alternatively, a specific combination of the spin axis orientation and the nucleus activity pattern might have kept the line position and shape fairly constant over the rotation cycle. Therefore, we limit our further discussion to the periodic variability of the production rate, which is independent of the line position variability.

\subsection{Methods}

Performing the periodicity analysis we generally followed the classical approach of Drahus \& Waniak (2006), where a constant frequency $f$ of the periodicity in the input time series is being determined. Although they also introduced a dynamical approach, where an evolution of the frequency with time $\mathrm{d} f / \mathrm{d} t$ is simultaneously searched for, we did not apply it to our data.
It has been well established, both in theory (e.g. Samarasinha et al. 1986; Jewitt 1999; Gutiérrez et al. 2002) and through observations (e.g. Belton \& Drahus 2007; Drahus \& Waniak 2006, and references therein), that the cometary nuclei, especially those that are small and active, can substantially change their rotation periods on a time scale of a single perihelion passage. Using the simple model of Drahus \& Waniak (2006) we estimated that the expected change of the rotation frequency of SW3-C during our campaign, regardless of the frequency itself, is about $0.04 \mathrm{~h}^{-1}$ (Appendix C.2). In the rotation phase profile for the middle frequency this corresponds to the shift of the first and last data point of more than a full rotation cycle, making the classical periodicity analysis completely irrelevant. At the same time their $d y$ namical approach, naturally accounting for this effect, would be ideal.

Nevertheless, we limited our investigation to the classical analysis only - although it seems incorrect at the moment - due to the insufficient quality of our data for the dynamical approach. Drahus \& Waniak (2006) have successfully tested the routine using simulations with a $S / N$ of about 3 , i.e. comparable with their best observing results (where $S / N$ is defined as half of the difference between maximum and minimum of the phase profile over the standard deviation of the points about their smooth representation), and using 128 synthetic data points, i.e. the same number as observed. On the other hand, the additional parameter may lead to the failure of this approach when the $S / N$ is worse and/or the number of input data points is smaller (Waniak \& Drahus, in preparation). In our case the number of measurements is very similar, but the $S / N$, being below 2 for half of the considered period solutions (further discussed in Sect. 5.3, particularly in Fig. 8), does not guarantee sufficient reliability of the dynamical investigation. Nevertheless, we show in Appendix C.2 that in contrast to our expectations - the analysis done within the framework of a constant periodicity approximation is probably justifiable in our case.

We performed the periodicity analysis using two different methods: the Phase Dispersion Minimization, originally proposed by Stellingwerf (1978), and generalized by Drahus \& Waniak (2006) in order to take statistical weights of individual data points into account (hereafter WPDM, where "W" indicates weighting), and the $\chi^{2}$ minimization using a sum of harmonics fitted to the data, that also accounts for the statistical weights of the individual measurements (hereafter HF, which stands for harmonics fit). Both methods return a variance ratio $\Theta$ which measures the quality of a phase profile at given frequency $f$. Generally, the lower the value of $\Theta$ is, the better the profile it indicates. The working parameters of WPDM are the number of bins and the number of covers (denoted as $N_{\mathrm{b}}$ and $N_{\mathrm{c}}$ respectively) and the only parameter of HF is the number of frequencies used for fitting (denoted as $N_{\mathrm{f}}$ ).

The error on the frequency $f$ of a given periodicity solution was estimated using the Monte Carlo approach, in a similar way as the error on line position (see Sect. 3). As the reference for simulations we used a smooth counterpart of the original phase profile, which we calculated using the HF method. (Note that we used HF for two different reasons: as an independent periodicitysearch routine, and in order to obtain a noiseless representation of a given phase profile.) The smooth profile was then disturbed with a large number of noise realizations, which we produced assuming a Gaussian noise distribution with a variance given by the square of the data point error. The simulated noisy phase profiles were analyzed with the same method and setting which originally had returned the solution whose error was under study. As the investigated error we adopted a standard deviation of the 


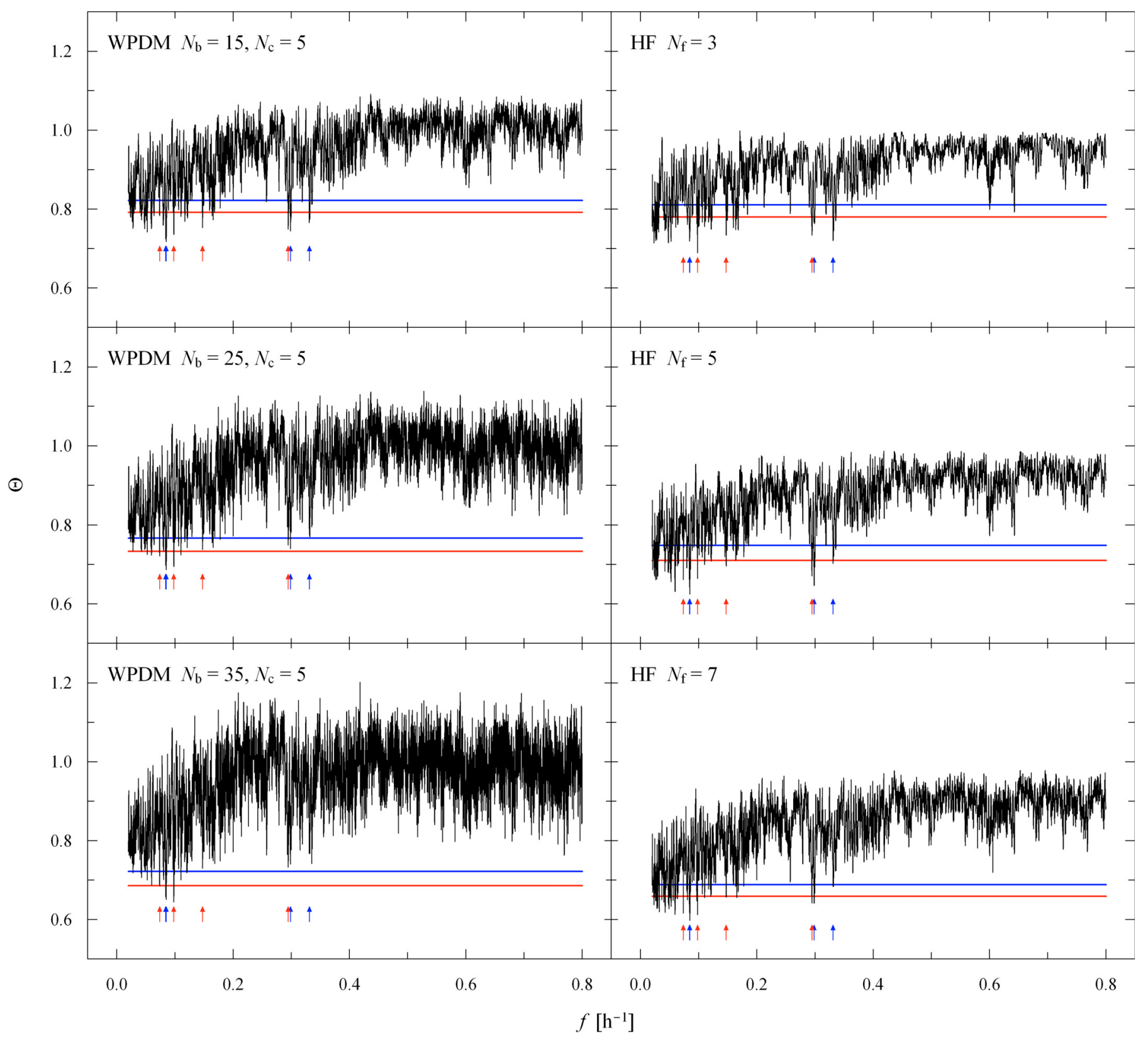

Fig. 7. Selected results from the search for periodicity in the normalized HCN production rates $Q_{0}$. The left panels show the periodograms obtained with the WPDM algorithm and the right panels with the HF method. The horizontal lines indicate the confidence levels of $75 \%$ (blue) and $95 \%$ (red). Positions of the eight best solutions (see Table 2) are indicated by the arrows: red for those from the system and blue for the others. Solutions B and C are barely resolved in this figure.

values from the simulations, calculated with respect to the actual frequency of the investigated solution. Note that such an approach naturally converts the uncertainties of the data points in the input time series into a standard error of periodicity (Drahus \& Waniak 2006).

Moreover, we also retrieved the probabilities that any phase profile - characterized by a certain value of $\Theta_{0}$ - does not result entirely from noise. Such confidence levels were derived also using the Monte Carlo method. Again, we simulated a number of noise realizations, taking however a constant reference curve this time. Then, they were analyzed using both methods with given settings, yet only global minima of $\Theta$ were retrieved. This made it possible to constrain individual statistics $N\left(\Theta<\Theta_{0}\right)$ for each method and setting, which provided the confidence levels for $\Theta_{0}$.

\subsection{Results}

Both methods were used to investigate the time series of our individual production rates $Q_{0}$, normalized to $r=1 \mathrm{AU}$ using the best-fit heliocentric dependence (see Sect. 4.2). We scrutinized the range of frequencies $f$ from 0.02 to $0.8 \mathrm{~h}^{-1}$ (corresponding to the periods $P$ from 1.25 to $50 \mathrm{~h}$ ), with a step size of $0.00002 \mathrm{~h}^{-1}$. As the statistical weights of the individual measurements we used the inverse squares of their errors. The WPDM algorithm was run with $N_{\mathrm{b}}$ ranging from 15 to 35 , and $N_{\mathrm{c}}=5$, whereas the HF method was used with $N_{\mathrm{f}}$ ranging from 3 to 7 . The most relevant periodograms were found for $N_{\mathrm{b}}=25$ (corresponding to 5 measurements per bin on average) and $N_{\mathrm{f}}=5$. This is because when working with noticeably lower values of these parameters, the methods do not return the whole set of existing solutions, whereas when using much higher values they do not provide any 
Table 2. Possible solutions for periodicity and their properties.

\begin{tabular}{|c|c|c|c|c|c|c|c|c|c|}
\hline Solution & $\begin{array}{c}f_{\mathrm{WPDM}^{a}} \\
{\left[\mathrm{~h}^{-1}\right]}\end{array}$ & $\begin{array}{l}f_{\mathrm{HF}^{b}}^{b} \\
{\left[\mathrm{~h}^{-1}\right]}\end{array}$ & $\begin{array}{c}f^{c} \\
{\left[\mathrm{~h}^{-1}\right]}\end{array}$ & $\begin{array}{l}P^{d} \\
{[\mathrm{~h}]}\end{array}$ & $M^{e}$ & $\begin{array}{c}f_{\mathrm{fit}^{f}} \\
{\left[\mathrm{~h}^{-1}\right]}\end{array}$ & $\sigma^{g}$ & $A^{h}$ & $S / N^{i}$ \\
\hline A & $0.07372 \pm 0.00004$ & $0.07370 \pm 0.00003$ & $0.07371 \pm 0.00004$ & $13.567 \pm 0.007$ & 4 & 0.07372 & 0.19 & 2.164 & 3.524 \\
\hline B & $0.08428 \pm 0.00008$ & $0.08424 \pm 0.00004$ & $0.08426 \pm 0.00006$ & $11.868 \pm 0.009$ & & & & 2.132 & 3.188 \\
\hline $\mathrm{C}$ & $0.08512 \pm 0.00008$ & $0.08504 \pm 0.00005$ & $0.08508 \pm 0.00007$ & $11.754 \pm 0.009$ & & & & 2.389 & 3.706 \\
\hline D & $0.09832 \pm 0.00007$ & $0.09826 \pm 0.00005$ & $0.09829 \pm 0.00006$ & $10.174 \pm 0.006$ & 3 & 0.09829 & 0.01 & 1.921 & 2.707 \\
\hline $\mathrm{E}$ & $0.14742 \pm 0.00016$ & $0.14758 \pm 0.00012$ & $0.14750 \pm 0.00014$ & $6.780 \pm 0.006$ & 2 & 0.14743 & 0.48 & 1.694 & 1.975 \\
\hline $\mathrm{F}$ & $0.29474 \pm 0.00024$ & $0.29480 \pm 0.00019$ & $0.29477 \pm 0.00021$ & $3.392 \pm 0.002$ & 1 & 0.29487 & 0.46 & 1.644 & 1.953 \\
\hline G & $0.29862 \pm 0.00022$ & $0.29860 \pm 0.00020$ & $0.29861 \pm 0.00021$ & $3.349 \pm 0.002$ & & & & 1.560 & 1.669 \\
\hline $\mathrm{H}$ & $0.33128 \pm 0.00015$ & $0.33112 \pm 0.00012$ & $0.33120 \pm 0.00014$ & $3.019 \pm 0.001$ & & & & 1.626 & 1.930 \\
\hline
\end{tabular}

${ }^{a} N_{\mathrm{b}}=25, N_{\mathrm{c}}=5 ;{ }^{b} N_{\mathrm{f}}=5 ;{ }^{c}$ unweighted-mean result from both methods with an effective error; ${ }^{d}$ corresponding period with an error; ${ }^{e}$ period multiple for the system, $M=1$ indicates the base of the system; ${ }^{f}$ frequency expected for an ideal system, given by an unweighted linear fit to the dependence of $f$ on $M ;{ }^{g}$ deviation of frequency $f_{\text {fit }}$ from $f$, given as a fraction of the error on $f ;{ }^{h}$ amplitude (see Sect. 5.1 and Fig. 8); ${ }^{i}$ signal-to-noise ratio (see Sect. 5.2 and Fig. 8).

additional solutions and are less stable due to the limited number of input measurements. For all the periodograms we retrieved the confidence levels of detection, simulating each time 1000 realizations of noise. The optimal periodograms are displayed in Fig. 7, along with the ones calculated for $N_{\mathrm{b}}=15$ and $N_{\mathrm{f}}=3$, as well as for $N_{\mathrm{b}}=35$ and $N_{\mathrm{f}}=7$, which we show for comparison.

The figure shows a good consistence between the results from the different methods and parameters. Although a number of solutions are present, only few of them are significant in a statistical sense. We selected eight most likely solutions, which were found in the following way: from the full set of periodograms we identified all the solutions characterized in at least one of them by the $\Theta$ value not higher than $5 \%$ above the minimum value in the considered periodogram. Next, we rejected all the solutions at $f<0.07 \mathrm{~h}^{-1}(P>14.3 \mathrm{~h})$ as they suffer from an insufficient phase coverage with our data. For each of the accepted solutions we retrieved the Monte Carlo error, considering separately the two positions from the two methods with the optimum settings (representing the same solution). As the reference profiles for the simulations we used smooth fits from the HF method with $N_{\mathrm{f}}=5$ for the solutions at $f<0.1 \mathrm{~h}^{-1}$ and $N_{\mathrm{f}}=3$ for those at $f>0.1 \mathrm{~h}^{-1}$, which provide the most realistic representations of the data. Onto each of them we superimposed 1000 noise realizations. As the final solutions we adopted the combined results from both methods. The positions and properties of the selected solutions are listed in Table 2, and the corresponding phase profiles are displayed in Fig. 8.

Half of these solutions, i.e. A, D, E, and F (see Table 2), form a system of period multiples which starts with solution $\mathrm{F}$. Some further multiples are also visible in the periodograms, although they do not satisfy our aforementioned requirements for a "good" solution. The system is so precise that all its constituents are found within a fraction of their errors from the expected frequencies. The reason that our periodograms are dominated by this structure is the very simple shape of the phase profile for its base frequency (solution F), which is nearly sinusoidal and features only one peak (what actually defines this solution as a base of the system). Therefore, it can be easily decomposed into the associated multiples, with slowly increasing complexity - each succeeding solution in the system features one additional peak in its phase profile, and all the peaks are images of the phase profile for the base frequency. Since the complexity of the profiles rises slowly, the system can be traced up to high period multiples (i.e. low frequencies), but of course at some point the profiles become overly complex, and the number of data points too low, thus the system vanishes. On the other hand, we cannot exclude the possibility that some multi-peak solution from this system is actually the correct one, which - just because of an unusually high symmetry - created the whole structure. However, in such a case, multiple, equiproductive, and equidistant (in the nucleocentric longitude) active sources are required, or an irregular nucleus with fairly uniform volatility must be assumed (if a double-peak solution $\mathrm{E}$ is considered) - but both scenarios are very specific, thus unprobable in our opinion. At the same time, all the independent solutions, unrelated to the system, are physically very plausible. The requirements of symmetries are not relevant to them, being in fact violated (otherwise the solutions would not be independent, being multiples of simpler periodicities). Therefore, the phase profiles are irregular, often featuring asymmetric multiple peaks, typically characterized by uneven amplitudes and/or unequidistant positions. Although these solutions are bases, they do not produce sharp systems of their multiples, due to rapid growth of the complexity (although in some cases first multiples are weakly visible).

Summarizing, we found a periodic oscillation of the reduced production rate $Q_{0}$ with the amplitude factor of 1.6-2.4 and $S / N$ of 1.7-3.7, depending on phasing. The most likely explanation of this effect is a variation of activity over the nucleus rotation cycle. However, our observations alone are insufficient to unambiguously point at the correct rotation period, which is due to the insufficient $S / N$ and large gaps in sampling. Possible problems which might have affected our analysis are discussed in Appendix C.

\subsection{Discussion}

In Sect. 5.3 we concluded that solutions A, E, and D (see Table 2), which are multiples of solution F, require a very symmetric distribution of the sublimation sources or a uniformly volatile nucleus (in the case of solution E), both scenarios being specific and thus unlikely in our opinion. This is supported by observations of the rotation phase profiles of coma in other comets, which are either simple one-peak sinusoids (cf. e.g. Schleicher \& Osip 2002; Biver et al. 2007) or irregular (cf. e.g. Drahus \& Waniak 2006; Manfroid et al. 2007). Although sometimes it is difficult to choose between a single-peak phase profile or its double-peak first multiple (e.g. Schleicher et al. 1991; Schleicher \& Osip 2002), to our knowledge a symmetric multipeak shape has never been unambiguously claimed. 
A\&A 510, A55 (2010)

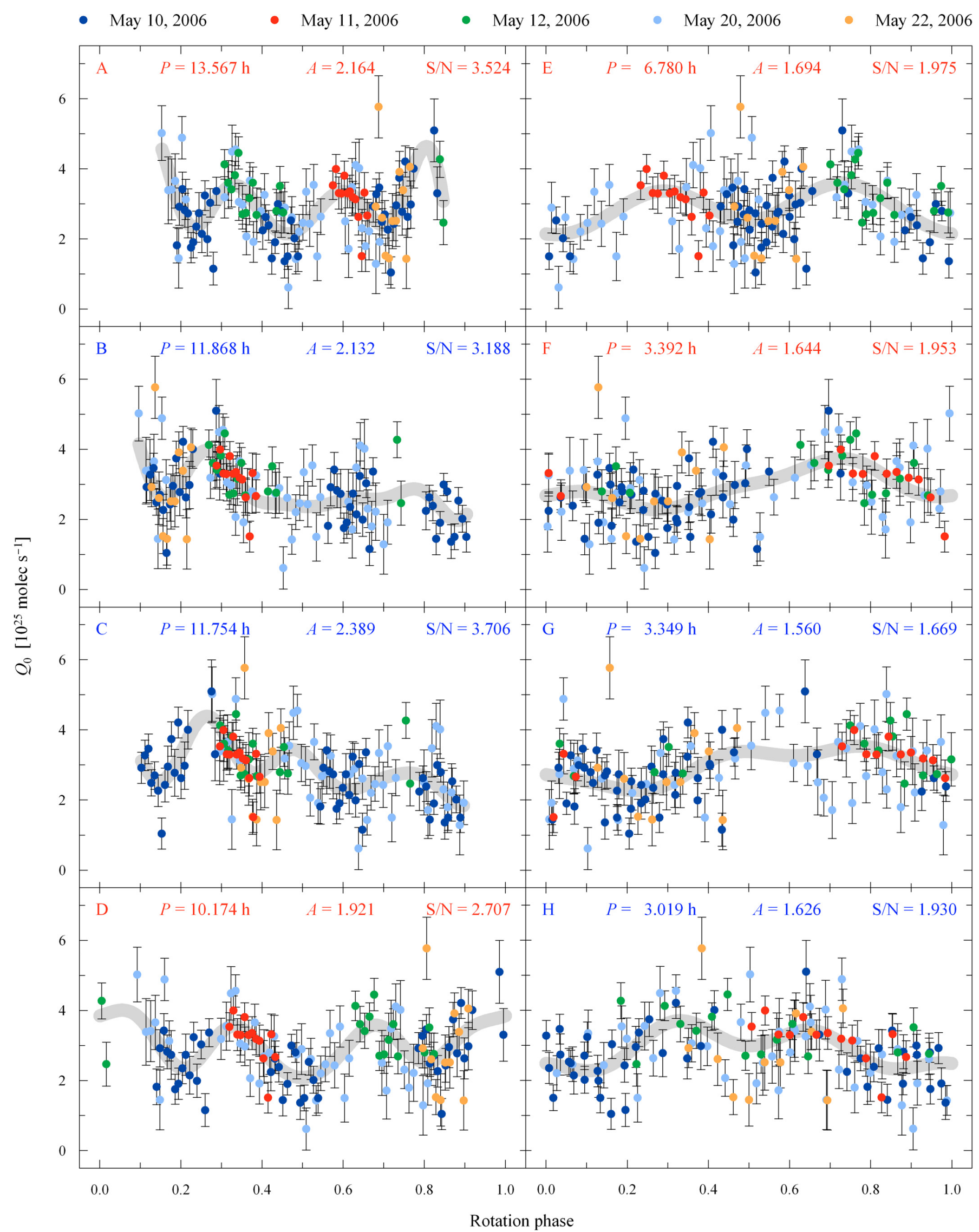

Fig. 8. Phasings of the normalized HCN production rates $Q_{0}$ according to the eight best solutions (see Table 2) with periods $P$. The red and blue fonts indicate the system and the other solutions respectively. The smooth fits (gray lines) were used as the reference profiles to calculate the errors of the discussed solutions. They also provide us with the amplitudes $A$ and enable calculation of the $S / N$. 
Table 3. Other determinations of the rotation period $P$ of SW3-C.

\begin{tabular}{|c|c|c|c|}
\hline Mid time (UT) & $P[\mathrm{~h}]$ & Method & Reference \\
\hline May 2,2000 & $\begin{array}{l}2.8 \\
3.2 \\
5.1\end{array}$ & $R$-band photometry of the inner coma & $\begin{array}{l}\text { Farnham (2001), } \\
\text { Farnham (priv. comm.) }{ }^{a}\end{array}$ \\
\hline April 3, 2006 & $\geq 3.8$ & $R$-band photometry of the inner coma & Waniak (priv. comm.) $)^{b}$ \\
\hline April 10, 2006 & $3.2 \pm 0.2$ & HST bare-nucleus equivalent photometry & $\begin{array}{l}\text { Toth et al. (2006), } \\
\text { Toth et al. (priv. comm.) }\end{array}$ \\
\hline 7,2006 & $\begin{array}{r}8.8 \pm 0.3 \\
13.2 \pm 0.3 \\
27.2 \pm 0.3\end{array}$ & Dust and CN coma morphology & Storm et al. (2006) \\
\hline May 12, 2006 & $>7$ & Doppler broadening of radar signal & $\begin{array}{l}\text { Nolan et al. (2006), } \\
\text { Nolan et al. (priv. comm.) }\end{array}$ \\
\hline
\end{tabular}

${ }^{a}$ Single-peak solutions, amplitude of $0.3 \mathrm{mag}$, aperture radius of $3^{\prime \prime} ;{ }^{b}$ amplitude of 0.03 mag, aperture radius of $6 " ;{ }^{c}$ double-peak solution, amplitude of $0.6 \mathrm{mag}$.

However, an argument supporting the multi-peak phase profiles may also be raised. Sekanina (2005) showed that on September 6, 1995 two fragments (i.e. B and E) broke off from SW3-C, and then on November 2, 1995 fragment F additionally disconnected. Provided that SW3-C did not experience further splitting, one may expect one major active region on its nucleus related to fragment $\mathrm{B}$, and two weaker ones, associated with the smaller fragments $\mathrm{E}$ and $\mathrm{F}$, interfering with the original activity pattern on the rest of the body. It is not clear, however, if the post-break-up active sources, created a decade before, survived until our campaign in 2006: although the comet was still exceptionally active (see Sect. 4.3), it has been continually losing its excessive productivity since it broke up (Boehnhardt 2004). Moreover, one should bear in mind that the real activity pattern does not necessarily correspond well to the observed phase profile (see Appendix A.5).

An important argument regarding the true rotation period comes from the analysis of two additional data sets, presented in Appendix C.5. The analysis clearly supports the shortest-period solutions, which are the most stable against distortions of the original data set. On the other hand, in Appendix C.4 we show that the noise-induced solutions occur preferentially at the highest frequencies, which is an argument supporting a longer rotation.

It is clear that we cannot choose the correct rotation period based on our observations alone. So far, a wide range of rotation periods was proposed for SW3-C, which we list in Table 3. Note that the middle value from Storm et al. (2006), which is $13.2 \pm 0.3 \mathrm{~h}$, is similar to our solution A. However, it is the fourth period-multiple in the system, so we consider this solution as unlikely for the reasons presented earlier in this section.

Furthermore, the rotation period of $3.2 \pm 0.2 \mathrm{~h}$, originally inferred by Toth et al. (2006) and later refined by Toth et al. (priv. comm.), is consistent with our three shortest-period solutions (i.e. F, G, and $\mathrm{H}$ - see Table 2). These solutions are plausible, and they are supported by several other arguments presented in the previous paragraphs. Assuming that the rotation period by Toth et al. (priv. comm.) and any of the three shortestperiod solutions were indeed correct at their epochs, we derive in Appendix C.2 additional interesting properties of SW3-C. We show there that the fraction of the total outgassing, which was effectively accelerating or decelerating the rotation, was below $0.23 \%$. This is much lower compared to comet Tempel 1 , which we attribute to the exceptionally large active fraction of the nucleus of SW3-C (see Sect. 4.3). Moreover, we estimated, that the rotation frequency did not change during our campaign by more than $0.0063 \mathrm{~h}^{-1}$, which validates our approximation of the constant rotation period for the three shortest-period solutions, and excludes the other ones from our list.

Overall, despite the disagreement with some period estimations, we suggest that the rotation period of comet SW3-C during our campaign was between 3.0 and $3.4 \mathrm{~h}$, and that it was fairly constant. That means that SW3-C is most probably the fastest rotator among the cometary nuclei identified to date (cf. e.g. Samarasinha et al. 2004). We cannot, however, choose between $P=3.392 \mathrm{~h}, 3.349 \mathrm{~h}$, and $3.019 \mathrm{~h}$ (solutions $\mathrm{F}, \mathrm{G}$, and $\mathrm{H}$ respectively) as we find them equally likely.

The arguments supporting fast and stable rotation of SW3-C cautiously suggest that originally the comet had been fragmented due to a large centrifugal force. Furthermore, it is entirely possible that the mother comet had been rotating even faster shortly before the split than did SW3-C during our campaign. That is because a fraction of the total angular momentum was taken away by the smaller fragments during the break-up, and it is likely that SW3-C has been slowly spinning down since then (otherwise it should have broken up again), which is, however, unimportant on the time scales comparable to the duration of our campaign. The hypothesis of a slow spin-down may be additionally supported if the shortest period from those found by Farnham (priv. comm.) for the half-way epoch, $P=2.8 \mathrm{~h}$, is the correct one, and if the intermediate period by Storm et al. (2006) can be explained as a fourth multiple of the real period. In such a case, our solution $\mathrm{F}$ (base of the system), which we found at $P=3.392 \mathrm{~h}$, being the one consistent with most of the other determinations, should be considered as the most likely at our epoch. But at the moment this is only a speculation, since we cannot be sure about the rotation period of the mother comet 10 years before, and we have no information about its properties, such as bulk tensile strength and density, and shape, which would enable us to calculate the critical rotation period for such a body (Davidsson 1999, 2001).

On the other hand, the fact that SW3-C was stable against the rotational disruption is very informative concerning its properties. Using the model of Davidsson (2001), and taking the suggested effective radius of $0.5 \mathrm{~km}$ (see Sect. 1) and the bulk density of $600 \mathrm{~kg} \mathrm{~m}^{-3}$ (as found for comet Tempel 1; cf. A'Hearn et al. 2005) along with the rotation period of $3.392 \mathrm{~h}$ (solution F) and the minimum axis ratio of 1.8 (Toth et al. 2006) 
as one extreme, we calculated that the bulk tensile strength of SW3-C must be of at least $14 \mathrm{~Pa}$. On the other extreme, taking the rotation period of $3.019 \mathrm{~h}$ (solution $\mathrm{H}$ ) and the actual axis ratio of 3 , tentatively suggested by Toth et al. (2006), we obtained the required bulk tensile strength of at least $45 \mathrm{~Pa}$. Both results are in agreement with the value of $50 \mathrm{~Pa}$, which was found by Belton et al. (2005) as likely for a cometary nucleus.

\section{Summary and conclusions}

Comet SW3 was observed from the SMT between May 1 and 22, 2006. Here we focused on the observations of $\mathrm{HCN}$ from five nights with good observing conditions.

Using the night-averaged spectra we found clear evolution of the line position with phase angle, which is characteristic for solar-stimulated activity. The same spectra provided us with the model-dependent coma expansion velocity of $0.8 \pm 0.1 \mathrm{~km} \mathrm{~s}^{-1}$, which is typical for a comet at this heliocentric distance. The average model-dependent production rate at $r=1 \mathrm{AU}$ was $(2.70 \pm 0.09) \times 10^{25}$ molec s $^{-1}$ (with about $20 \%$ of absolute uncertainty), showing that the nucleus of SW3-C was exceptionally active. The calculated production rate is similar to the corresponding results obtained by other authors, although some discrepancies are present. In this work we do not address the problem of these discrepancies, because such a study would be premature at the moment, keeping in mind that the results from several other (sub-) millimeter, IR, and optical campaigns have not been published yet. When they are available, several interesting questions potentially may be answered. Particularly, what is the real slope of the long-term evolution of the HCN production rate for this comet? Is HCN the only important parent of CN? Are the IR results consistent with the (sub-) millimeter ones? And if not, what is the underlying reason for this discrepancy?

We have shown that for several physical reasons parent molecules are very good candidates for tracing the rotation of active comets. Indeed, we found a strong (about a factor of 2) short-term variability of the HCN production, significantly exceeding the observing noise. It is most likely associated with the rotation of the nucleus of SW3-C. This means that great care must be taken when interpreting production rates from short observing sessions of parent molecules within the inner coma (cf. the day-to-day variability of SW3 noticed by Dello Russo et al. 2007). Several possible solutions for periodicity are found. Three of them, falling in the range 3.0-3.4 h, are consistent with the determination by Toth et al. (priv. comm.), and we consider them as the most realistic from our list. The apparent stability of the rotation period suggests that only a very small fraction of the total outgassing, perhaps $0.2 \%$ or less, was effectively accelerating or decelerating the nucleus rotation. This contrasts with the significantly higher efficiency of this process in comet Tempel 1, which is plausibly explained by the much larger active fraction of the nucleus of comet SW3-C. The period of 3.0-3.4 h is unusually short for a cometary nucleus, tentatively suggesting too fast rotation as the reason for the original splitting. However, since we cannot trace the spin evolution of SW3-C back to the moment of break-up, and a reliable threshold for rotational disruption of the mother comet cannot be calculated, our result cannot be considered as a solid proof of this scenario. On the other hand, the observed stability of SW3-C against rotational disruption is informative concerning the bulk tensile strength of the nucleus, which we found to be of at least 14-45 $\mathrm{Pa}$.

The rotation period of a comet shortly before it splits would indicate the reason for break-up much better than the period determined post-factum. Therefore, following Drahus \& Waniak (2006), we postulate monitoring of the spin states of a possibly large number of active comets, which may spin-up or -down due to torques induced by non-radial outgassing. It is then only a question of time as to when a prediction of rotational break-up will become possible for a certain object, giving us a definite test of this concept.

We demonstrated that such research can be done by applying standard periodicity analysis to a time series of parent molecule production rates, calculated from the inner coma in a standard way. Indeed, it is probably the most natural "language" for studying the rotation of active comets, as it automatically provides good estimates for some parameters of outgassing, such as the production rate itself and a sublimation velocity, which are essential in constraining physical models of the accelerating spin of the nucleus.

Acknowledgements. The SMT is operated by the Arizona Radio Observatory, Steward Observatory, University of Arizona. We thank the SMT staff for their support during the observations. We also thank Dr. Wacław Waniak (Astronomical Observatory of the Jagiellonian University) who had codeveloped some software used for this study, Prof. Klaus Jockers (Max-PlanckInstitut für Sonnensystemforschung) for his valuable suggestions, and our referee, Dr. Jacques Crovisier (Paris Observatory), for his valuable remarks.

\section{References}

A’Hearn, M. F., Millis, R. L., Schleicher, D. G., Osip, D. J., \& Birch, P. V. 1995, Icarus, 118,223

A'Hearn, M. F., Belton, M. J. S., Delamere, W. A., et al. 2005, Science, 310, 258 Altenhoff, W. J., Baars, J. W. M., Wink, J. E., \& Downes, D. 1987, A\&A, 184, 381

Baars, J. W. M., \& Martin, R. N. 1996, Rev. Mod. Astr., 9, 111

Baars, J. W. M., Martin, R. N., Mangum, J. G., McMullin, J. P., \& Peters, W. L. 1999, PASP, 111, 627

Belton, M. J., \& Drahus, M. 2007, BAAS, 39, 498

Belton, M. J., Meech, K. J., A'Hearn, M. F., et al. 2005, Space Sci. Rev., 117, 137

Biver, N., Bockelée-Morvan, D., Crovisier, J., et al. 1999, AJ, 118, 1850

Biver, N., Bockelée-Morvan, D., Colom, P., et al. 2002a, Earth, Moon, and Planets, 90, 5

Biver, N., Bockelée-Morvan, D., Crovisier, J., et al. 2002b, Earth, Moon, and Planets, 90, 323

Biver, N., Bockelée-Morvan, D., Boissier, J., et al. 2007, Icarus, 187, 253

Bockelée-Morvan, D., \& Crovisier, J. 1985, A\&A, 151, 90

Bockelée-Morvan, D., \& Crovisier, J. 1987, The role of water in the thermal balance of the coma, in Proceedings of the Symposium on the Diversity and Similarity of Comets, ed. E. J. Rolfe \& B. Battrick (ESA SP-278, Noordwijk, The Netherlands), 235

Bockelée-Morvan, D., Padman, R., Davies, J. K., \& Crovisier, J. 1994, Planet. Space Sci., 42, 655

Bockelée-Morvan, D., Biver, N., Moreno, R., et al. 2001, Science, 292, 1339

Bockelée-Morvan, D., Crovisier, J., Mumma, M. J., \& Weaver, H. A. 2004a, The Composition of Cometary Volatiles, in Comets II, ed. M. C. Festou, H. U. Keller, \& H. A. Weaver (Tucson: Univ. of Arizona press), 391

Bockelée-Morvan, D., Biver, N., Colom, P., et al. 2004b, Icarus, 167, 113

Boehnhardt, H. 2004, Split Comets, in Comets II, ed. M. C. Festou, H. U. Keller, $\&$ H. A. Weaver (Tucson: Univ. of Arizona press), 301

Boehnhardt, H., \& Kaufl, H. U. 1995, IAU Circ., 6274

Boissier, J., Bockelée-Morvan, D., Biver, N., et al. 2007, A\&A, 475, 1131 Crovisier, J., Biver, N., Bockelée-Morvan, D., et al. 1995, IAU Circ., 6227

Davidsson, B. J. R. 1999, Icarus, 142, 525

Davidsson, B. J. R. 2001, Icarus, 149, 375

Dello Russo, N., Vervack, R. J., Weaver, H. A., et al. 2007, Nature, 448, 172

Drahus, M., \& Waniak, W. 2006, Icarus, 185, 544

Farnham, T. L. 2001, BAAS, 33, 1047

Feaga, L. M., A'Hearn, M. F., Sunshine, J. M., Groussin, O., \& Farnham, T. L. 2007, Icarus, 191, 134 
M. Drahus et al.: The HCN molecule as a tracer of the nucleus rotation of comet 73P-C

Fray, N., Bénilan, Y., Cottin, H., Gazeau, M.-C., \& Crovisier, J. 2005, Planet. Space Sci., 53, 1243

Gutiérrez, P. J., Ortiz, J. L., Rodrigo, R., López-Moreno, J. J., \& Jorda, L. 2002, Earth, Moon, and Planets, 90, 239

Hale, A., Kobayashi, J., \& Morris, C. S. 1995, IAU Circ., 6234

Haser, L. 1957, Bull. Acad. R. Sci. Liege, 43, 740

Henry, F., Bockelée-Morvan, D., Crovisier, J., \& Wink, J. 2002, Earth, Moon, and Planets, 90, 57

Huebner, W. F., Keady, J. J., \& Lyon, S. P. 1992, Ap\&SS, 195, 1

Jewitt, D. 1999, Earth, Moon, and Planets, 79, 35

Jorda, L., \& Gutiérrez, P. 2000, Earth, Moon, and Planets, 89, 135

Kadota, K., Jaeger, M., Galad, A., et al. 2000, IAU Circ., 7534

Kobayashi, H., Kawakita, H., Mumma, M. J., et al. 2007, ApJ, 668, L75

Kutner, M. L., \& Ulich, B. L. 1981, ApJ, 250, 341

Lamy, P. L., Toth, I., Fernández, Y. R., \& Weaver, H. A. 2004, The sizes, shapes, albedos, and colors of cometary nuclei, in Comets II, ed. M. C. Festou, H. U. Keller, \& H. A. Weaver (Tucson: Univ. of Arizona press), 223

Lamy, P. L., Toth, I., A'Hearn, M. F., Weaver, H. A., \& Jorda, L. 2007, Icarus, 187,132

Lis, D. C., Keene, J., Young, K., et al. 1997, Icarus, 130, 355

Magee-Sauer, K., Mumma, M. J., DiSanti, M. A., Dello Russo, N., \& Rettig, T. W. 1999, Icarus, 142, 498

Manfroid, J., Hutsemékers, D., Jehin, E., et al. 2007, Icarus, 187, 144

Michałowski, T. 1988, Acta Astron., 38, 455

Milam, S. N., Apponi, A. J., Ziurys, L. M., \& Wyckoff, S. 2006, IAU Circ., 8702

Morris, C. S., Kronk, G. W., Garradd, G., et al. 1995, IAU Circ., 6246
Müller, H. S. P., Schlöder, F., Stutzki, J., \& Winnewisser, G. 2005, J. Mol. Struct., 742,215

Nolan, M. C., Harmon, J. K., Howell, E. S., et al. 2006, BAAS, 38, 504

Pickett, H. M., Poynter, R. L., Cohen, E. A., et al. 1998, J. Quant. Spectrosc. \& Rad. Transfer, 60, 883

Samarasinha, N. H., A'Hearn, M. F., Hoban, S., \& Klinglesmith, D. A. 1986, $\mathrm{CN}$ jets of Comet P/Halley - Rotational properties, in ESA Proc. of the 20th ESLAB Symp. on the Exploration of Halley's Comet, ESA SP-250, 487

Samarasinha, N. H., Mueller, B. E. A., Belton, M. J. S., \& Jorda, L. 2004, Rotation of cometary nuclei, in Comets II, ed. M. C. Festou, H. U. Keller, \& H. A. Weaver (Tucson: Univ. of Arizona press), 281

Schleicher, D. G. 2006, Cent. Bureau Elect. Telegram, 491

Schleicher, D. G., \& Osip, D. 2002, Icarus, 159, 202

Schleicher, D. G., Millis, R. L., Osip, D. J., \& Birch, P. V. 1991, Icarus, 94, 511

Schloerb, F. P., Kinzel, W. M., Swade, D. A., \& Irvine, W. M. 1986, ApJ, 310, L55

Scotti, J. V., Galad, A., Boehnhardt, H., et al. 1996, IAU Circ., 6301

Sekanina, Z. 2005, Int. Comet Quarterly, 27, 225

Stellingwerf, R. F. 1978, ApJ, 224, 953

Storm, S., Samarasinha, N., Mueller, B., et al. 2006, BAAS, 38, 504

Toth, I., Lamy, P., \& Weaver, H. A. 2005, Icarus, 178, 235

Toth, I., Lamy, P., Weaver, H., et al. 2006, BAAS, 38, 489

Ulich, B. L., \& Haas, R. W. 1976, ApJS, 30, 247

Villanueva, G. L., Bonev, B. P., Mumma, M. J., et al. 2006, ApJ, 650, L87

Weaver, H. A. 2006, BAAS, 38, 484

Woodney, L. M., Owen, T. C., \& Fernández, Y. R. 2003, IAU Circ., 8239

Pages 14 to 22 are available in the electronic edition of the journal at http : / /www . aanda . org 


\section{Appendix A: Validation of the model and parameters}

Here we estimate the uncertainty of the conversion of the line area $\int T_{\mathrm{mB}} \mathrm{d} v$ into the corresponding production rate $Q$, introduced by the simplifying assumptions of our model and by the uncertainties of the adopted model parameters (Sect. 4.1). We performed the calculations using the values calculated or assumed in Sects. 4.1 and 4.2, i.e., $\left\langle Q_{0}\right\rangle=2.70 \times 10^{25}$ molec s$^{-1}$, $T=80 \mathrm{~K}$, and $v_{\exp }=0.8 \mathrm{~km} \mathrm{~s}^{-1}$. Whenever the water production rate was needed, we used $1.2 \times 10^{28}$ molec s$^{-1}$, which we consider as a mean-diurnal value during our campaign. It is based on the result by Schleicher (2006), who estimated the production of water from his optical observations of $\mathrm{OH}$, but we scaled his result to $r=1 \mathrm{AU}$ (through a power law with an exponent of -4 , which we consider as a "textbook" value; cf. Sect. 4.3). This is consistent with the results from Villanueva et al. (2006) and Dello Russo et al. (2007), based on direct observations of $\mathrm{H}_{2} \mathrm{O}$ in IR, which are scattered around the adopted value when normalized in the same way (marginally or not important for the latter). Note that the IR-based production rates are likely to be snapshot values whereas the $\mathrm{OH}$-based result is presumably a mean-diurnal production rate (cf. Sects. 5.1 and 6).

First, working with the model itself, we investigated how much flux is contributed to our observations by the molecules at different nucleocentric distances $\rho$. Such dependence is entirely controlled by the beam pattern: in our model the total number of molecules in a thin shell centered at the nucleus is independent of the shell radius (if the thickness is fixed), and also the light emission properties of the molecules are identical throughout the coma. We also calculated cumulative flux contributions from the nucleocentric distances below a certain radius. Both profiles are presented in Fig. A.1. In the final step, with the aid of these profiles, we investigated the effects introduced individually by each of the assumptions, along with the input parameters, which we present in the following sections.

\section{A.1. Was the coma optically thin?}

Let us consider an optical depth $\tau=0.25$ as small enough to validate treatment of the coma as optically thin. Then, assuming for a moment the coma to be completely stationary, this criterion is satisfied at $\rho \geq 100 \mathrm{~km}$ for the $J(3-2)$ transition and at $\rho \geq$ $150 \mathrm{~km}$ for the $J(4-3)$ one. However, Fig. A.1 shows that even in the extreme case (May 10, 2006), when the size of the beam was smallest (see Table 1), and the optical depth largest, only $13 \%$ of the molecules were within the optically thick regime.

In fact the cometary coma is not stationary but rather expands rapidly. Therefore, molecules along a column have different velocity components along the line of sight, and due to different Doppler shifts, the emission from the molecules are slightly misaligned in frequency. For this reason, the optical depth along a column of an expanding coma is smaller than for a static coma with the same number density - hence the net impact on our derivations is $\ll 13 \%$.

\section{A.2. Were LTE conditions present throughout the coma?}

We convolved the model flux contributions (Fig. A.1) with the nucleocentric profiles of the non-LTE to LTE occupancy ratios of the $J=3$ and $J=4$ rotational levels. We found that the determined production rates are overestimated by $6.5-13.5 \%$ (depending mostly on the beam size). We adopted the non-LTE

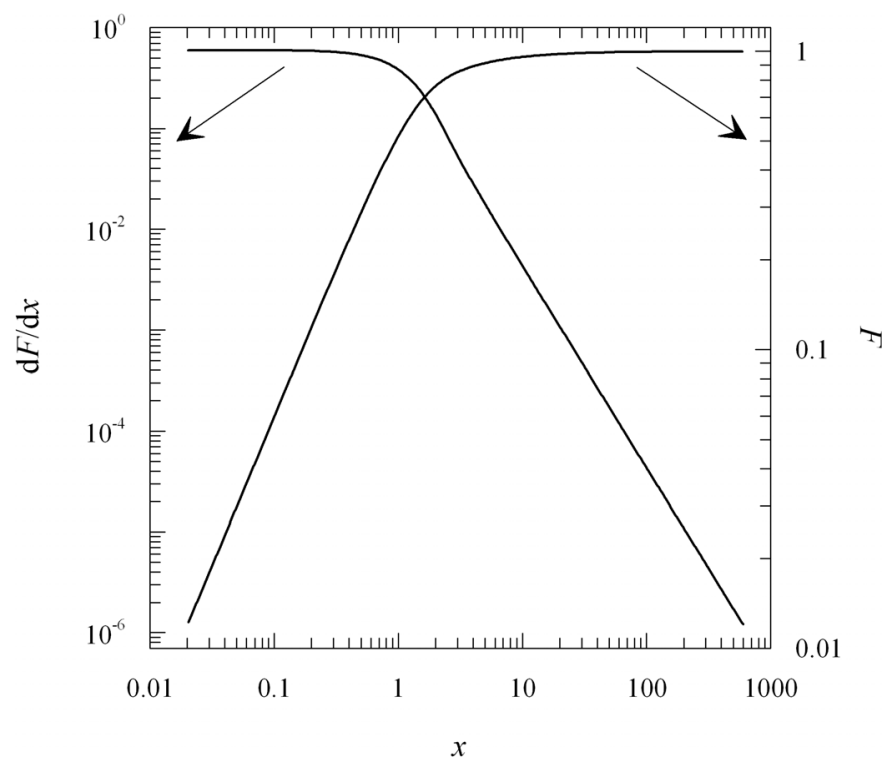

Fig. A.1. Flux in the model coma as observed by our model beam. The left vertical scale concerns the relative flux contribution $\mathrm{d} F / \mathrm{d} x$, generated in a shell of a unit thickness and the nucleocentric radius $x$. The right vertical scale concerns the cumulated relative flux $F$, produced in a sphere with the nucleocentric radius $x$. The $x$ quantity is a relative length, measured in units of the beam radius at half-power.

distribution of the rotational levels at $1 \mathrm{AU}$ from the Sun from Bockelée-Morvan et al. (2004a), but we scaled the nucleocentric distance to keep the water density profile consistent with SW3-C, which was an order of magnitude less productive than assumed in their model. Although the adopted distribution was calculated for the (constant) kinetic temperature of $T=50 \mathrm{~K}$, this inconsistence is probably negligible.

The analysis shows, that determination of the production rates assuming the Boltzmann distribution of the energy levels was justifiable in our case; by no means, however, can this fact be identified with the LTE conditions present throughout the observed coma, which we do not address here.

\section{A.3. Was the photodissociation process negligible?}

A convolution of the model flux contributions (Fig. A.1) with an exponential decay of $\mathrm{HCN}$ gives $2.5-4.5 \%$ underestimation of the determined production rates (depending on the beam size). We used for this test the quiet-Sun $\mathrm{HCN}$ photodissociation rate of $1.26 \times 10^{-5} \mathrm{~s}^{-1}$ at $r=1 \mathrm{AU}$ (Huebner et al. 1992). The error introduced by this assumption is very small because the comet approached the Earth so closely that we observed the inner coma only (half-power radius of the beam was about $1000 \mathrm{~km}$ ). The photodissociation process, however, played an important role at much larger nucleocentric distances, since the characteristic photodissociation scale-length for HCN was about $60000 \mathrm{~km}$.

\section{A.4. Were the molecules isotropically ejected from the nucleus?}

Comet SW3-C was sublimating preferentially in (roughly) the Sunward direction, which is perfectly clear from the evolution of the line position with phase angle, visible in the night-averaged spectra (Sect. 3). Isotropic outgassing is additionally refuted by the single-peak shape of the night-averaged spectra, as isotropic models, such as ours, predict an obvious central dip when the 


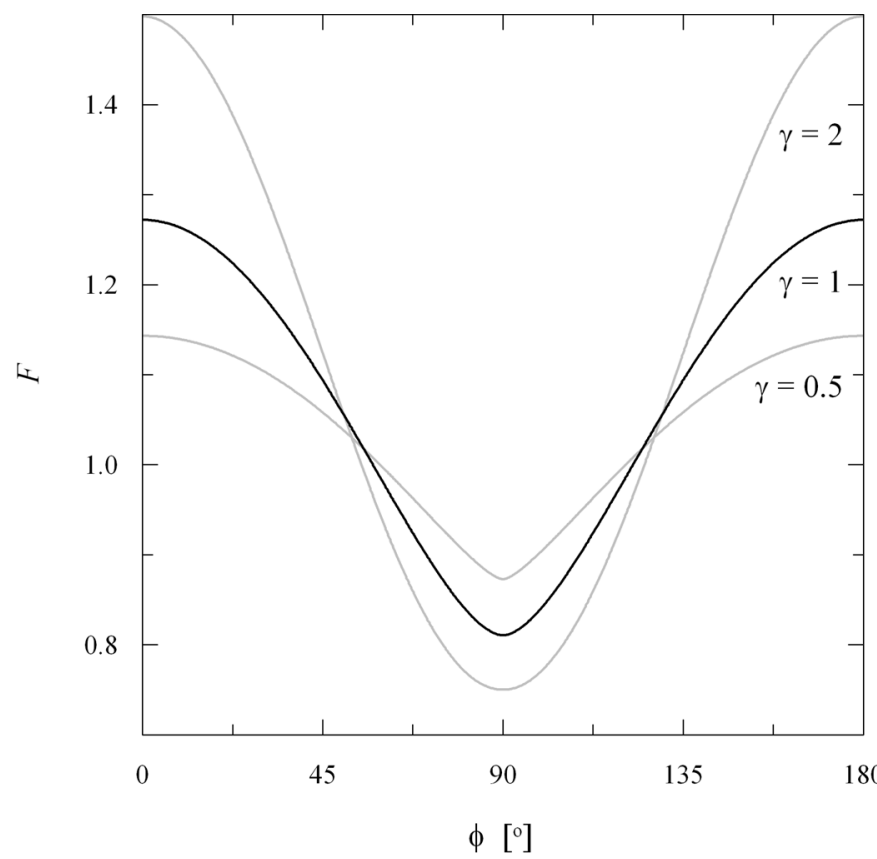

Fig. A.2. Dependence of the relative model flux $F$ on phase angle $\phi$ for different exponents $\gamma$ (see text).

coma is larger than the beam. Anisotropic, pro-solar outgassing is understandable, since it is the Sun that controls the temperature distribution over the nucleus. Therefore, there is no question of whether or not the molecules were isotropically ejected from the nucleus, but rather of how important the violation of this assumption is, while determining the production rate using our model.

Let us first consider separately the impact of an anisotropic outgassing on the derived production rates at two extreme geometries: a sublimation restricted to the plane perpendicular to the line of sight, and restricted to the plane parallel to the line of sight, where both planes cross the nucleus. For simplicity we assume here that outgassing is constant in time. In the former situation, there is no influence in the calculated production rates, i.e. the total flux observed by the beam leads to an unambiguous retrieval of this quantity regardless of the angular profile of the local sublimation rates (that may not be constant over the nucleus due to e.g. its dependence on the insolation angle). However, the latter scenario permits either an excess or a deficit of the observed flux with respect to the isotropic sublimation (at the same global rate), depending on a preferred direction of outgassing. An excess of the flux appears when the molecules are being preferentially ejected along the line of sight, thus many of them (i.e. more than for an isotropic sublimation) accumulate in the beam for a long time. On the other hand, if the molecules are being preferentially ejected in a direction perpendicular to the line of sight, many of them leave the beam rapidly, resulting in a deficit of the observed flux. In consequence, the calculated production rate is respectively over- and underestimated, if a model assuming an isotropic sublimation is used for the retrieval. Considering a comet whose nucleus is a uniformly volatile plain sphere whose outgassing is entirely controlled by the zenith angle of the Sun, it is intuitively clear that the largest underestimation of the production rate should be expected when the comet is at the phase angle $\phi=90^{\circ}$, whereas the highest overestimation should occur at $\phi=0^{\circ}$ and $180^{\circ}$.

This agrees with our quantitative analysis which is presented in Fig. A.2. It was obtained in the framework of our model, generalized however to account for the anisotropy of the outgassing. As the activation function we used $\cos ^{\gamma} z$, where $z$ is local zenith angle, and $\gamma=1$ (though we also show the results for $\gamma=0.5$ and $\gamma=2$ for comparison). If $\gamma=0$ is used, the model reduces itself to the standard isotropic version. For simplicity we assumed that the molecules are ejected in the direction strictly perpendicular to the nucleus surface. Although this formally implies no collisions and $T=0 \mathrm{~K}$ (in a thermodynamical sense), such "artifacts" of the model are not very important while investigating the influence of geometric projections. The obtained result is very general, e.g. independent of the beam size and pattern, as long as the other assumptions of our model are satisfied (e.g. negligible photodissociation).

Unfortunately, it is very difficult to model an anisotropic outgassing in a realistic, time-dependent way (cf. Appendix A.5), thus the isotropic model of Haser (1957) is the choice of almost every author (see e.g. A'Hearn et al. 1995; Bockelée-Morvan et al. 2004a). We followed this approach - even though it is not very realistic - for the obvious reason of simplicity, and also to keep the basic consistence with most of the other published results (note that the photodissociation process, naturally tackled by the Haser model, is negligible in our case - see Appendix A.3). For our observations of comet SW3-C, which was around $\phi=90^{\circ}$ in that time (see Table 1), this may result in about a $20 \%$ systematic underestimation of the production rates (see Fig. A.2). In spite of this, the self-consistence of the values should be practically unaffected, thanks to the fairly constant phase angle, which varied only $\pm 15^{\circ}$ around the minimum of this dependence (see Table 1).

\section{A.5. Was the production rate constant in time?}

We analyze the heliocentric evolution (Sect. 4) and the shortterm variability (Sect. 5) of the HCN production, although we had determined the rates under the assumption that they are constant. This assumption, which greatly simplifies our model making it time-independent, is however well satisfied in both cases, considering each process as a sequence of isolated states, such as the production is constant within each particular state, although it varies from state to state.

The characteristic time scale for this problem, calculated in the framework of our model, is only about $45 \mathrm{~min}$, and after this time most of the molecules (73-87\% depending on the beam size) would have left the beam (encircled by its radius at halfpower). This is a clear advantage of the exceptionally close approach of SW3 to the Earth (see Table 1), making our beam unusually small at the comet distance. Provided that the production rate evolved on a time scale significantly longer than $45 \mathrm{~min}$, and bearing in mind that the individual exposures (4-5 $\mathrm{min}$ ) never exceeded this characteristic time scale, the obtained single spectra indeed can be considered as snapshots of a steady-state coma.

Obviously the heliocentric evolution of the production rate happens on a time scale much longer than 45 min (Sect. 4.2). However, is the same argumentation valid to justify our analysis of the short-term variation (Sect. 5.3)?

First, a rotation phase profile of the production rate which one observes may be noticeably different from the outgassing pattern intrinsic to the nucleus (cf. Biver et al. 2007). Generally, it is delayed in phase, its amplitude is decreased and shape deformed, which is caused by the non-zero beam size and nonzero exposure, that imply collecting the flux from molecules released at different moments of time - thus different rotation phases or even cycles. Most importantly, however, the periodicity of the observed phase profile remains unchanged thus can be 
directly linked to the rotation period of the nucleus. The faster the molecules leave the beam, and the slower the nucleus rotates, and the shorter the individual exposures are - the closer the observed phase profile is to the intrinsic one. However, to determine only the rotation period correctly, it is already sufficient to satisfy the three aforementioned conditions to be constant in time at any values.

In such a case, a physical interpretation of the phase profile (e.g. determining an instantaneous total production rate, locating and comparing sources of activity) may be done upon full nonsteady-state modeling. Consider for example a nucleus which does not sublimate at all at some rotation phase, but the line is still visible due to the emission from the molecules released earlier. In such a case one will determine a substantial production rate if a steady-state model, such as ours, is used for the retrieval. But even if a non-steady-state modeling is performed, or if we deal with a truly intrinsic phase profile, such an interpretation may still be problematic. This is because the phase profile is entirely controlled by superposition of instantaneous ejections from the active regions at a given rotation phase, so we can barely resolve the individual sources at similar nucleocentric longitudes as well as those over which the insolation does not change noticeably over the rotation cycle (though in both cases they can be isolated by modeling the spectral line shape and/or by imaging). Therefore, when investigating a phase profile of the production rate, one should rather refer to the effective active sources.

We are convinced that the discussed effects of flux processing do not affect our observations significantly, and hence the observed phase profile is close to the effective intrinsic activity pattern of the nucleus (regardless of the slightly varying exposure and the noticeably varying beam size). This is because both the characteristic time scale for a significant loss of the molecules from the beam and the individual exposures were shorter than the time scale of any clear feature stimulated by the nucleus rotation (see Fig. 8). Interestingly, this justification applies to all the eight phase profiles, because those that are highly structured are found at low frequencies, and the complexity decreases fairly linearly with increasing frequency.

Note also a comparison of properties of the phase profiles for parent coma vs. daughters and dust presented in Sect. 5.1.

\section{A.6. Were the expansion velocity and the temperature of the molecules constant in space and time? Were they equal to the adopted values?}

Let us restrict this analysis to the nucleocentric distances $\rho=$ 200-5000 km, from where about $80 \%$ of the model flux contribution comes. For a comet at $r=1 \mathrm{AU}$ with the production rate of water as estimated for SW3-C, in this range the nucleocentric profile of the outflow velocity should be practically constant at about $0.9 \mathrm{~km} \mathrm{~s}^{-1}$ (see e.g. Bockelée-Morvan \& Crovisier 1987). This is very close to the value of $0.8 \pm 0.1 \mathrm{~km} \mathrm{~s}^{-1}$, which we derived from the line widths in the night-averaged spectra (see Sect. 4.1), and which is consistent with the determinations for other comets around the same heliocentric distance (cf. e.g. Biver et al. 1999, 2002a).

However, we are conscious that this result may be affected by the several simplifications of our model. Particularly, if most of the outgassing goes permanently in one direction, the corresponding spectral line may be very narrow regardless of the outflow velocity, and thus may result in significant underestimation of the velocity if a model assuming an isotropic sublimation (such as ours) is used to fit the line width. For a rotating
Table A.1. List of published HCN rotational temperatures $T_{\text {rot }}$ based on IR spectroscopic observations of SW3-C.

\begin{tabular}{ccccc}
\hline \hline $\begin{array}{c}\text { Date UT } \\
(2006)\end{array}$ & $\begin{array}{c}r \\
{[\mathrm{AU}]}\end{array}$ & $\begin{array}{c}\text { Coma extract } \\
{[\mathrm{km} \times \mathrm{km}]}\end{array}$ & $\begin{array}{c}T_{\text {rot }} \\
{[\mathrm{K}]}\end{array}$ & Reference $^{a}$ \\
\hline April 7 & 1.270 & $101 \times 420$ & $52_{-10}^{+15}$ & {$[1]$} \\
April 16 & 1.194 & $75 \times 312$ & $53_{-10}^{+18}$ & {$[1]$} \\
May 14 & 1.000 & $25 \times 102$ & $77 \pm 2$ & {$[2]$} \\
May 15 & 0.995 & $26 \times 105$ & $88 \pm 3$ & {$[2]$} \\
\hline
\end{tabular}

${ }^{a}$ [1] Villanueva et al. (2006), [2] Dello Russo et al. (2007).

nucleus with discrete active source(s), the situation may be improved by the flux processing (see Appendix A.5), which simulates isotropization of the coma. This is because such a narrow line would be drifting in frequency as the gas radial velocity changes over the rotation cycle, and hence the processed line would be broader. Our night-averaged spectra are definitely subjected to this effect due to their long effective exposures, however, it is not likely to significantly isotropize the coma as we did not find any hint of the line position variability due to the nucleus rotation (see Sect. 5.1). Moreover, since the observations were obtained in fairly "frozen" geometry (see Table 1), even if we used a campaign-averaged spectrum, the Sunward anisotropy (cf. Sect. 3) would remain barely reduced by the processing. As we concluded in Appendix A.4, it is very difficult to model an anisotropic outgassing in a realistic, time-dependent way, therefore we determined the expansion velocity in a standard manner, which is easy to implement and which ensures basic consistence with most of the other published results.

The model by Bockelée-Morvan \& Crovisier (1987) also suggests that the kinetic temperature of the parent coma has a nearly constant nucleocentric profile at about $15 \mathrm{~K}$ in our case. The very low model temperature is a direct result of the cooling process induced by the adiabatic expansion of gas, with only very weak heating by highly energetic photodissociation products, whose number was simply too low for comet SW3-C to efficiently drive the process. However, e.g. Lis et al. (1997) determined a (rotational) temperature equal to $73 \mathrm{~K}$ for comet Hyakutake at very similar geo- and heliocentric distances, and using a similar observing facility (beam radius $400-800 \mathrm{~km}$ depending on the transition frequency), whereas the same model suggests $T<40 \mathrm{~K}$ already accounting for the 10 times higher productivity of that comet. This suggests that the temperature yield of this model should be considered as a robust lower limit for the mean gas temperature within our beam.

Additional constraints on the $\mathrm{HCN}$ temperature come from IR observations of SW3-C (Table A.1). As expected, the temperature was rising while the heliocentric distance and the size of the sampled coma were decreasing. Unfortunately, the helio- and nucleocentric dependencies cannot be decoupled, nor are any of these measurements compatible with our beam size. However, since the temperatures by Dello Russo et al. (2007) were determined at very similar heliocentric distances, our best guess is that they are at the high end of what is reasonable to expect for our observations of SW3-C. We realize that they were sensitive only to the high-temperature region close to the nucleus, whereas our beam sampled the more distant colder gas too; however, the warmer gas, heated by collisions with the photodissociation products further from the nucleus, should also contribute to our beam if the process was efficient (see the characteristic nucleocentric scalelengths for the adiabatic cooling and the photolytic heating in e.g. Bockelée-Morvan \& Crovisier 1987). 


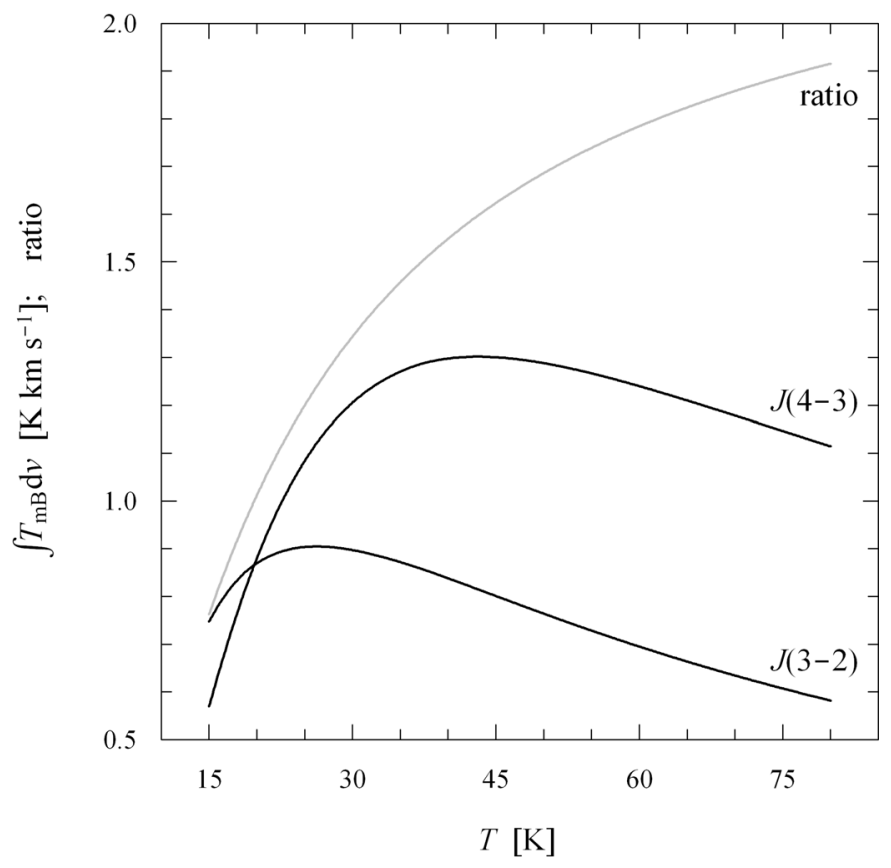

Fig. A.3. Dependence of the line area $\int T_{\mathrm{mB}} \mathrm{d} v$ on the coma temperature $T$ (assumed to be constant) for both considered transitions (black lines). It was derived with our model for the $\mathrm{HCN}$ production rate $Q=2.7 \times 10^{25}$ molec s$^{-1}$, the geocentric distance $\Delta=0.1 \mathrm{AU}$, and the beam properties as of the SMT (see Sect. 2). The line ratio is also displayed (gray line).

Temperature determinations from other molecules and for fragment B (also reported by other authors), although educative, unfortunately do not remove the ambiguity of this discussion.

The observed $J(4-3)$-to- $J(3-2)$ line-area ratio (cf. Fig. 3) is also suggestive about the temperature, although it cannot be used to measure it directly. One problem is that we did not observe both lines simultaneously, hence their ratio is influenced by the nucleus rotation if different rotation phases were sampled on different nights (cf. Sect. 5), and, to a smaller extent, by the slight change in observing geometry (see Table 1). Another problem is that the beam size was different for each of the observed transitions (cf. Table 1), hence the line ratio is influenced by the nucleocentric temperature profile (see before), and also because of a possible drop of the main-beam efficiency at the higher frequency (though undetected - see Sect. 2). Nevertheless, using the night-averaged spectra from May 10 and 11, 2006 (see Fig. 3), we computed the ratio of $1.34 \pm 0.05$, which - in LTE conditions - suggests a gas temperature of only $30 \pm 2 \mathrm{~K}$ (see Fig. A.3). However, taking the aforementioned effects into account it is clear that this estimation should be considered as another lower limit on the temperature.

Taking all the arguments into account we eventually adopted $T=80 \mathrm{~K}$, which may be, however, slightly overestimated.

Nevertheless, for both monitored transitions we checked with our model how the line area $\int T_{\mathrm{mB}} \mathrm{d} v$ depends on the temperature $T$, and the result is presented in Fig. A.3. It shows that if for example we took the temperature as low as $45 \mathrm{~K}$, the production rate obtained from the $J(3-2)$ transition would be $38 \%$ lower, and $14 \%$ lower from the $J(4-3)$ one. Therefore, if the temperature were lower than adopted, then the production rates we report in this work would be slightly overestimated, and the heliocentric dependence would be overly steep (Sect. 4.2). On the other hand, the influence of the uncertainty on the temperature is too weak to affect our periodicity analysis (Sect. 5), which is fairly stable against much larger changes of the heliocentric reduction (see Appendix C.5).

The facts that the temperature probably varies noticeably with the nucleocentric distance (cf. the profiles in Bockelée-Morvan \& Crovisier 1987, for higher production rates), and with direction (cf. analysis of this effect for the production rate in Appendix A.4) are not very important here, since its constant value is a model concept only, aimed at optimum retrieval of the production rates. Therefore it should be considered as an effective temperature for a given beam rather than a precisely defined thermodynamic property of the coma. The expansion velocity is less sensitive to these effects, which are practically negligible compared to the problems discussed in the second paragraph of this section.

The heliocentric evolution of the coma expansion velocity and of the temperature must be practically negligible on the time scale of our campaign, which is due to the excellent stability of the observing geometry (see Table 1). Similarly, we ensured that the uncertainty about the temperature is negligible for the determination of the expansion velocity.

We acknowledge that in this discussion we consider the rotational temperature to be the same as the kinetic temperature hence we directly compare the observational measurements of the former with the model predictions for the latter. However, this identity is true only under LTE, which was not necessarily well satisfied in our case (see Appendix A.2).

Considering the determined production rates as isotropic, it is clear that all the other model assumptions do not affect them significantly. Note that the stability of our instrument was about $20 \%$ (see Sect. 2), and the statistical error of an individual measurement is also $20 \%$ on average. Furthermore, the estimated deficit of our production rates resulting from the assumptions discussed in Appendices A.1 and A.3 cancels to some extent the excess resulting from the assumption discussed in Appendix A.2 and from the adopted coma temperature discussed in Appendix A.6. Thus, the overall systematic error introduced by the deviation of our model from reality should be lower than $20 \%$, and the self-consistence of our production rates much better, justifying the use of this simple approach.

\section{Appendix B: Validation of the heliocentric correction}

The heliocentric evolution of the production rate is superimposed onto the short-term variability due to the rotation of the cometary nucleus. The determined heliocentric dependance may be different from the real one if the rotation phase of the nucleus is sampled irregularly. To evaluate the uncertainty introduced by this effect on our mean-diurnal $\mathrm{HCN}$ production rate at $r=1 \mathrm{AU}$, and on its heliocentric evolution, we assumed a simple sinusoidal rotation phase profile, coupled with a power-law dependance on heliocentric distance. Then the production rate $Q$ at the time $t$ and the heliocentric distance $r$ is given by:

$Q=\left(1+\frac{A-1}{A+1} \sin \left(2 \pi\left(t-t_{0}\right) f\right)\right) Q_{0}\left(r / r_{0}\right)^{n}$,

where $Q_{0}$ is the mean-diurnal production rate at $r=r_{0}=1 \mathrm{AU}$, $n$ is an exponent of the power-law heliocentric dependence, $A$ is 


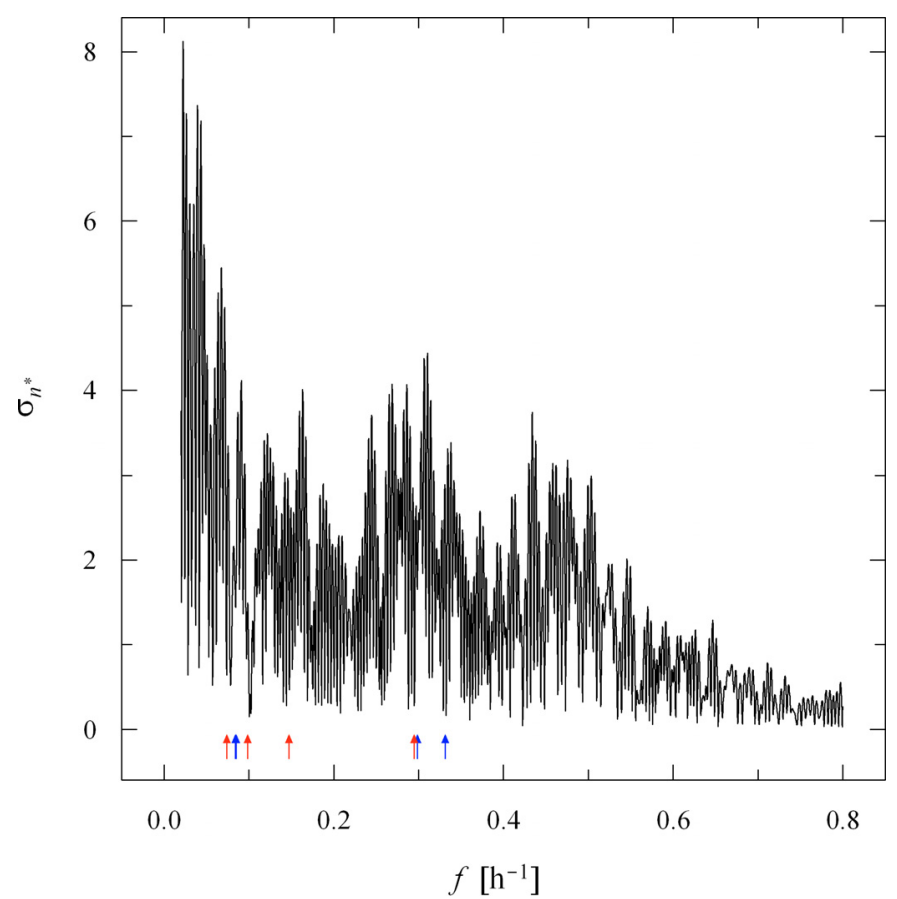

Fig. B.1. Standard deviation among the reconstructed power-law exponents, $\sigma_{n^{*}}$, as a function of the rotation frequency $f$. The simulated production rates were calculated from Eq. (B.1). Positions of the eight best solutions (see Table 2) are indicated by the arrows: red for those from the system and blue for the others, though solutions $\mathrm{B}$ and $\mathrm{C}$ are unresolved.

the ratio between maximum and minimum production rate during one rotation cycle, $f$ is the rotation frequency, and $t_{0}$ is the moment of the zero rotation phase.

We used $A=2$, as typically found in our analysis (see Sect. 5.3), and $n=-8.2$ as obtained from our data (see Sect. 4.2). For the frequency $f$ between 0.02 and $0.8 \mathrm{~h}^{-1}$, and the unknown zero-phase moment $t_{0}$ ranging between 0 and $1 / f$, we calculated the model production rates according to Eq. (B.1), using the moments of time $t$ of our observations. Then we determined $Q_{0}^{*}$ and $n^{*}$ from a power-law fit to such artificially created data, as we did for our HCN measurements (cf. Sect. 4.2).

Generally, the reconstructed quantities $Q_{0}^{*}$ and $n^{*}$ are different from the input ones, $Q_{0}$ and $n$, because the heliocentric reduction cannot take into account the rotation term, that is unknown at that point. We found that the measured $Q_{0}^{*}$ tends to be slightly lower than the real $Q_{0}$. Its mean value $\left\langle Q_{0}^{*}\right\rangle$, calculated from the complete range of the zero-phase moments $t_{0}$, is between 1.3 and $2.9 \%$ lower than $Q_{0}$, depending on the frequency $f$. The standard deviation of $Q_{0}^{*}$ around $\left\langle Q_{0}^{*}\right\rangle$, measuring how strongly the determined $Q_{0}^{*}$ depends on the zerophase moment $t_{0}$ at a given frequency $f$, is found between 0.1 and $17.3 \%$, with a median of only $2.9 \%$. Generally, the error introduced on $Q_{0}^{*}$ by the influence of the nucleus rotation is smaller than those from the other error sources, which are discussed in Appendix A.

The mean value of the reconstructed power-law exponent, $\left\langle n^{*}\right\rangle$, is always equal to the real $n$, regardless of the frequency $f$. This is because the variations average out when varying $t_{0}$ over a full rotation period. The standard deviation $\sigma_{n^{*}}$, as a function of frequency $f$, is shown in Fig. B.1. It shows that it is possible to obtain from our data $n^{*}$ which is very different from the real $n$, especially for lower frequencies $f$, but the discrepancy is very sensitive to the actual $f$. Moreover, we investigated the probability of obtaining $n^{*} \geq-4$ (which we consider as a "textbook" exponent; cf. Sect. 4.3), strongly differing from the real $n=-8.2$, and found it to be equal to $2.2 \%$. The same analysis, limited to the rotation periods in the range 3.0-3.4 $\mathrm{h}$ (as the most likely rotation period of SW3-C - see Sect. 5.4), yielded the probability of $3.6 \%$. On the other hand, if we would have assumed $n=-4$, then the probabilities of deriving $n^{*} \leq-8.2$ are $2.1 \%$ and $3.8 \%$ for the overall and restricted period ranges respectively.

Interestingly, the discussed functions of the rotation frequency $f$, such as the standard deviations of the reconstructed $Q^{*}$ and $n^{*}$, as well as $\left\langle Q_{0}^{*}\right\rangle / Q_{0}$, do not depend on the actual input values of $Q_{0}$ and $n$. But since the distribution of $n^{*}$ around the assumed $n$ is slightly asymmetric, they cannot be interchanged while calculating probabilities, which is illustrated by our results shown in the previous paragraph.

Summarizing, a small possibility exists that the steep instantaneous slope of the heliocentric dependance, derived in Sect. 4.2, is an artifact. However, this is still unlikely to significantly affect the periodicity analysis, as in Appendix C.5 we show that the presence of the solutions between 3.0 and $3.4 \mathrm{~h}$ is stable against such large changes in the assumed heliocentric evolution of the production rate.

\section{Appendix C: Validation of the periodicity analysis}

The analysis of periodicity presented in Sect. 5.3 is clearly insensitive to the systematic uncertainties of the production rates introduced by the simplifications of our model, which we extensively discuss in Appendix A. There are, however, several other problems which might have potentially affected the analysis. We discuss them in this section, and present direct tests for the robustness of our results.

\section{C.1. Seasonal effects?}

Following the statistical approach of Drahus \& Waniak (2006), who adopted the routine of Michałowski (1988), we investigated the extent to which the orbital motions of the comet and Earth might have affected the observed periodic variability of the HCN production rate driven by the nucleus rotation (cf. Sect. 5.3). The results are summarized in Table C.1. They show that the changes in the observing geometry might have produced shifts of the rotation phase, but they are relatively small compared to the scatter of the individual data points. Furthermore, since such phase shifts increase roughly linearly with time, they are well compensated for by a (very small) adjustment of the rotation period, which is then called synodic (in contrast to the "real" one, so-called sidereal). Small phase shifts additionally suggest that the amplitude and shape of the phase profile were also stable during our observations.

\section{C.2. Angular acceleration of the nucleus?}

Small yet active cometary nuclei are subjected to rapid changes of their rotation periods (see Sect. 5.2). The effect is produced by torques which appear as a result of the outgassing in nonradial directions. Even though the torques may be small, and may largely cancel out, the effective residual torque is still expected to efficiently drive the process, and consequently to cause a measurable change in the rotation period on a time scale of a single apparition. 
Table C.1. Maximum possible phase shifts ${ }^{a}$.

\begin{tabular}{ccc}
\hline \hline Date UT $^{b}$ & \multicolumn{2}{c}{ Confidence level } \\
\cline { 2 - 3 } (May 2006) $^{\text {May }}$ & $75 \%$ & $95 \%$ \\
\hline 11.6885 & 0.004 & 0.009 \\
12.5524 & 0.008 & 0.016 \\
20.6222 & 0.040 & 0.083 \\
22.4882 & 0.047 & 0.100 \\
\hline
\end{tabular}

${ }^{a}$ Calculated with respect to the mid-point of the first observing run, May 10.4979, 2006 UT. The statistics were constrained from over 1 million spin axis orientations distributed uniformly in space. ${ }^{b}$ Middle moment of an individual observing run.

Several authors, e.g. Samarasinha et al. (1986), Jewitt (1999), and recently Drahus \& Waniak (2006), presented a very similar simple description of this effect. Using the notation of the last authors, an instantaneous rate of change in the rotation frequency $\mathrm{d} f / \mathrm{d} t$, can be described as:

$\mathrm{d} f / \mathrm{d} t=\frac{15}{16 \pi^{2}} \frac{v_{\text {subl }} Q_{\text {tot }}}{R^{4} \varrho} S$,

where $v_{\text {subl }}$ is the gas sublimation velocity, $Q_{\text {tot }}$ is the production rate of all gaseous species, $R$ is the effective radius of the nucleus, $\varrho$ is the nucleus bulk density, and $S$ is a dimensionless scaling factor which is the fraction of the total outgassing $Q_{\text {tot }}$ which effectively accelerates or decelerates the rotation.

Using this equation we estimated the magnitude of angular acceleration for SW3-C during our campaign. The sublimation of gas was characterized by $Q_{\text {tot }}=1.2 \times 10^{28}$ molec s$^{-1}$ (the adopted water production rate; see the beginning of Appendix A), and $v_{\text {subl }}=0.8 \mathrm{~km} \mathrm{~s}^{-1}$ (the expansion velocity of the HCN coma; see Sect. 4.1), and the nucleus by $R=0.5 \mathrm{~km}$ (Toth et al. 2005, 2006; Nolan et al. 2006), and $\varrho=600 \mathrm{~kg} \mathrm{~m}^{-3}$ (as found for comet Tempel 1; cf. A'Hearn et al. 2005). The least known parameter is $S$, which we assumed to be also the same as for Tempel 1. Since the nucleus of the latter was recently discovered to be slowly spinning up (Belton \& Drahus 2007), the rate of change in the frequency, measured in the Deep Impact photometry at the level of $7 \times 10^{-8} \mathrm{~h}^{-2}$, suggests $S=1.5 \%$, when linked with the other measured properties through Eq. (C.1). Finally, as the estimation for SW3-C we obtained $\mathrm{d} f / \mathrm{d} t=1.4 \times$ $10^{-4} \mathrm{~h}^{-2}$. Although it is a factor of 2000 stronger than measured in comet Tempel 1, it is not a surprise that comet SW3-C should be changing its spin much faster than the six-times-larger nucleus of comet Tempel 1 (cf. A'Hearn et al. 2005), bearing in mind the biquadratic dependence on the radius, and that the comets had comparable production rates during both campaigns (cf. Biver et al. 2007).

Integration of $\mathrm{d} f / \mathrm{d} t$ over the duration time of our campaign yields the expected change of the rotation frequency equal to $0.041 \mathrm{~h}^{-1}$, regardless of the frequency itself. Double integration gives the expected change of the rotation phase, which is equal to 1.51 when referred to the middle moment of the campaign. The latter shows that the classical approach (see Sect. 5.2) is irrelevant in such a case because the phase shift of the first and last data point is more than one full rotation cycle.

In spite of this expectation, an argument suggesting a fairly constant periodicity may also be raised. As we noted in Sect. 5.4, the rotation period of $3.2 \pm 0.2 \mathrm{~h}$, obtained by Toth et al. (priv. comm.) for April 10, 2006, is similar to our three shortestperiod solutions (i.e. F, G, and $\mathrm{H}$ - see Table 2). If both their result and any of these three solutions are indeed correct at their epochs, then the corresponding change of the rotation period between these two campaigns does not exceed $0.2 \mathrm{~h}$. This corresponds to $\mathrm{d} f / \mathrm{d} t \approx \pm 2.2 \times 10^{-5} \mathrm{~h}^{-2}$, and consequently yields the scaling factor of approximately $S \approx \pm 0.23 \%$; which is a factor of 6.5 lower than for comet Tempel 1, and hence the net torque in SW3-C was much less efficient. This may result from the exceptionally large active fraction of the nucleus of SW3-C, which was about a factor of 20 more volatile than the one of comet Tempel 1 (see Sect. 4.3 and especially Fig. 6). Jewitt (1999) shows that the scaling factor (which he calls a dimensionless moment arm and denotes as $k_{T}$ ) is inversely proportional to the square-root of number of active sources randomly distributed in azimuth. If therefore we interpret the difference in nucleus volatility as a difference in number of active sources, we will obtain the scaling factor ratio of both comets of approximately 4.5, which is comparable to what we derived before taking the simplicity of our argumentation into account.

This result should be now confronted with the duration of our campaign. The change of the rotation frequency is implied to be equal to $\pm 0.0063 \mathrm{~h}^{-1}$, and the change of the rotation phase equal to 0.23 when referred to the middle moment of the campaign. To satisfy the latter we need to assume that if the rotation period were not constant, the frequencies of the considered solutions (determined in the classical way) would correspond to the middle time of the campaign - which is, however, very likely, since the time arrangement of our data is rather symmetric, and the quality does not change too much with time. Normally, such phase shifts should visibly decrease the quality of the phase profiles. But our observations cluster at the beginning and at the end of the campaign, therefore the phase shifts are fairly constant within each group (although formally proportional to the square of time), and they are well compensated for by a (very small) adjustment of the rotation period. The residual phase shifts are then insufficient to affect the obtained periodicities, and introduce only some small and "safe" excess scatter.

Note that the low efficiency of the accelerating torque, and consequently, the small changes of the rotation parameters during our campaign, are still the upper limits. That is because any other rotation period, within the uncertainty range of the result by Toth et al. (priv. comm.), would be even closer to one of the three shortest-period solutions from our list - thus being in support of it and suggesting slower changes of the rotation period. At the same time the increased difference with respect to the other solutions would make them unlikely. That is because faster changes of the rotation period would be required, which would consequently make their reality (as detected in the classical way) questionable, though we acknowledge that in such case the scaling factor would be closer to its theoretically-predicted value. An indication towards one of our three shortest-period solutions would also fix the sign of the period changes: currently we find the spin-up and the spin-down scenarios equally possible as the rotation period by Toth et al. (priv. comm.) is almost exactly between the extreme solutions $\mathrm{H}$ and $\mathrm{F}$.

We conclude that our analysis, being done within the framework of a constant rotation period, is fully justifiable for the shortest-period solutions (i.e. F, G, and H). All the other solutions may be considered as realistic only under the assumption that the period found by Toth et al. (priv. comm.) is incorrect, and the rotation was sufficiently stable during our campaign. Otherwise, they must be regarded as artifacts, as the presence of any of them would require a very rapid evolution of the rotation period, making detection of such a solution strongly problematic, and actually requiring disruption of SW3-C, due to an 
overly fast rotation, shortly before the HST run, which was not observed.

\section{C.3. Changes of the spin axis orientation?}

Clearly, torques induced by outgassing may also excite the nucleus rotation and reorient the total angular momentum vector (cf. e.g. Gutiérrez et al. 2002; Samarasinha et al. 2004), producing changes of the spin axis orientation.

However, the possibility that the considered periodicities (Table 2) are affected or even completely generated by complex rotation instead of simple is very unlikely. In such a case the periods of rotation and precession must have been very close to a resonance, probably yielding a much longer apparent periodicity in our analysis. Only the $1: 1$ resonance would enable detection of the true period, although not distinguished as complex with our methods. But it is difficult to imagine a reason for such a (or even any) resonance, and even harder a comet rotating with a period much shorter than $3 \mathrm{~h}-$ as stability of the nucleus is then problematic (cf. e.g. Davidsson 1999, 2001). Moreover, if the obliquity of the instantaneous spin axis to the total angular momentum vector was not large or the period of precession was long compared to the duration of our campaign, and if the time scale for substantial reorientation of the total angular momentum vector was long, then the spin axis orientation could not change much during our campaign. Consequently, the true periodicity could be correctly detected with our methods, as the discussed effects would only increase scatter in the phase profiles.

Therefore, we investigated our phase profiles using this scatter as a tracer of the discussed effects. Taking into account the rms error of our reduced production rates $Q_{0}$, which is equal to $0.582 \times 10^{25}$ molec s$^{-1}$, we derived that $\Theta$ would be equal to 0.448 if the data points were scattered as suggested by the rms. In contrast, the three discussed periodicities are detected at $\Theta$ of only $0.641-0.785$ (cf. Fig. 7), corresponding to $20-32 \%$ excess of scatter (though noticeably lower than $49 \%$ for the unphased data). Thus, it may indeed hint at some small changes of the spin axis orientation or the observing geometry (discussed in Appendix C.1), but it may also result from some instrumental effects (see Sect. 2), and/or intrinsically imperfect repeatability of the sublimation pattern. In any case, the detected periodicity should be practically unaffected.

\section{C.4. Where do the noise-induced solutions preferentially occur?}

Another argument that should be considered in this discussion refers to the statistical properties of the noise-induced solutions (see Sects. 5.2 and 5.3). So far, by analyzing the number $N$ of solutions satisfying the condition $\Theta<\Theta_{0}$ (which is fairly independent of the frequency $f$ ), we showed that all the solutions listed in Table 2 are statistically significant (see the confidence levels in Fig. 7). Nevertheless, another statistic $N\left(f_{0}<f<f_{0}+\mathrm{d} f\right)$, indicating at which frequencies the noise-induced (global) solutions preferentially occur, provides an additional constraint on the robustness of our results. We present it in Fig. C.1.

As might have been expected, most of the noise-induced solutions can be found at high frequencies. This means that if some of the solutions obtained for our observational data were in fact induced by noise, they would be the shortest-period ones rather than the others, which are practically impossible to generate from noise at $f<0.15 \mathrm{~h}^{-1}$. On the other hand, one should

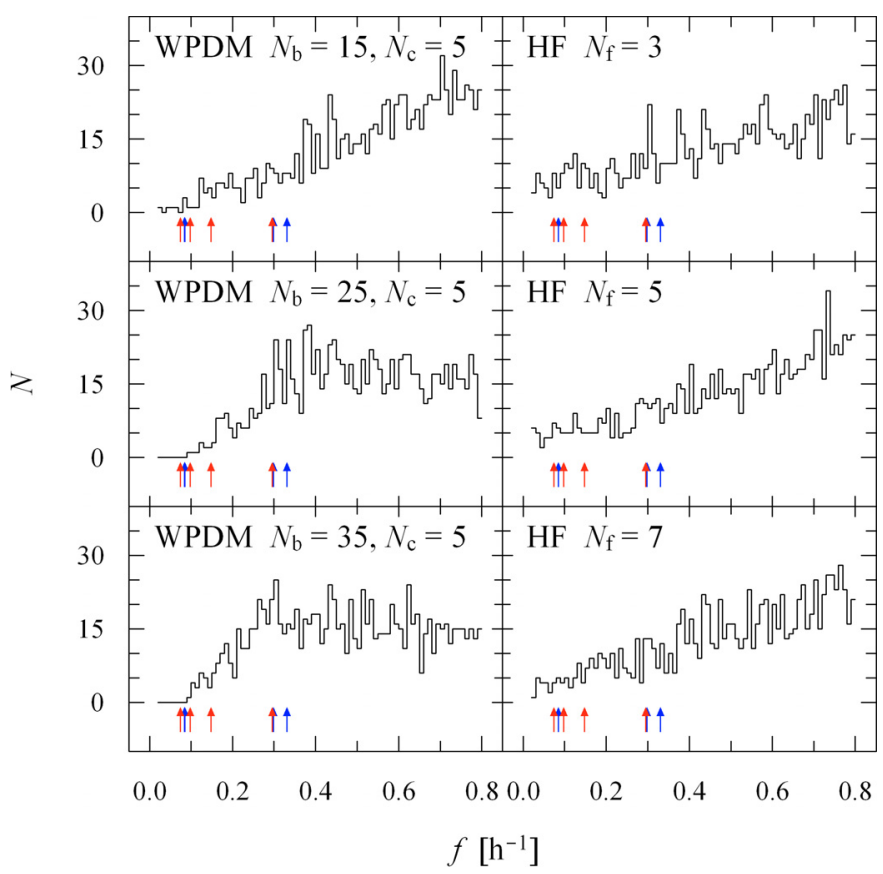

Fig. C.1. Histograms of the noise-induced solutions. Here $N$ indicates the number of simulations (from the total number of 1000) featuring global minima within a $0.01 \mathrm{~h}^{-1}$ frequency interval centered at $f$. Positions of the eight best solutions (see Table 2) are indicated by the arrows: red for those from the system and blue for the others, though solutions B and C are unresolved.

bear in mind that all the solutions we list were found at sufficiently low $\Theta$ to be certain that their presence is due to real short-term variability of the production rate. Therefore, the matter of the debate is only which of the phasings is the true one, but this ambiguity cannot be resolved with such an analysis.

\section{C.5. Analysis of two test data sets}

We also verified our results by performing the same periodicity analysis as before (cf. Sect. 5.3) for two additional data sets.

Data set A is based on the same AOS observations as used previously (cf. Sect. 2), but a different heliocentric trend was subtracted: we used a much shallower power law than before (cf. Sect. 4.2), with an exponent of -4 , which we consider as a "textbook" value (cf. Sect. 4.3).

Data set B was constrained from the spectra provided by the facility Forbes Filterbanks (FFB-A and FFB-B), which were working simultaneously with the AOS spectrometers. They have total bandwidths of about $1 \mathrm{GHz}$ with 1024 channels each (a resolution of $1000 \mathrm{kHz}$ ). These spectra were reduced in the same way as the AOS observations (cf. Sect. 2), and their own powerlaw heliocentric trend was subtracted (slightly steeper than the one calculated for the AOS observations - cf. Sect. 4.2). In theory, the meaningfulness of data set B is rather limited, because the observing noise comes mainly from the receiver, thus all the spectrometers should feature the same noise level and even pattern; in practise, however, this is not exactly true, because additional sources of noise also might have been present (cf. Sect. 2).

All the solutions we found before are also detected in these two tests, and most of them are again represented by the deepest minima (see Fig. C.2). However, two additional significant solutions are also present: one at $f=0.16569 \mathrm{~h}^{-1}$ in data set $\mathrm{A}$ 
M. Drahus et al.: The HCN molecule as a tracer of the nucleus rotation of comet 73P-C

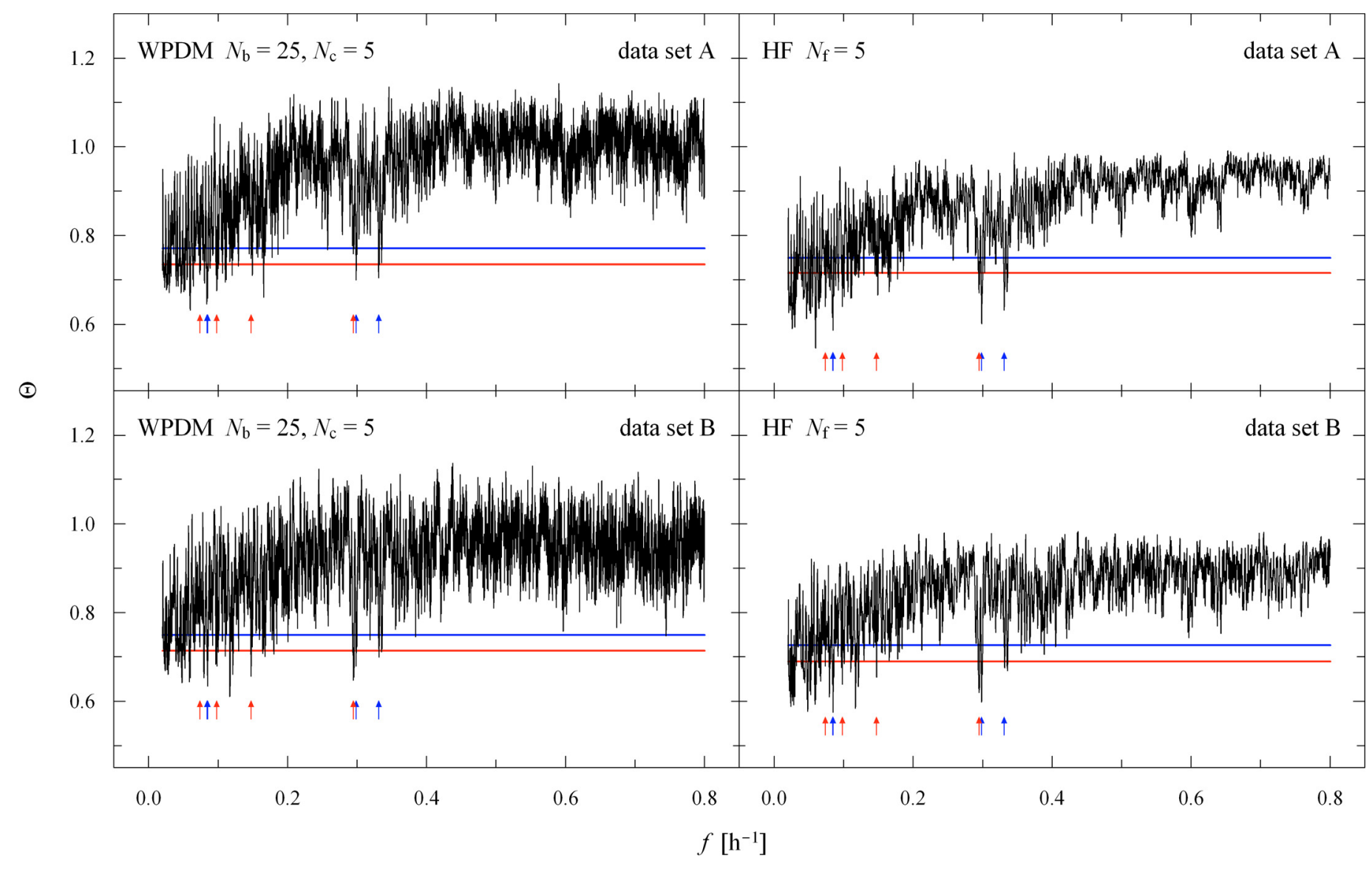

Fig. C.2. Periodograms for our two test data sets (described in the text) calculated with both methods. The horizontal lines indicate the confidence levels of $75 \%$ (blue) and $95 \%$ (red). Positions of the eight best solutions (see Table 2) are indicated by the arrows: red for those from the system and blue for the others. Solutions B and C are barely resolved in this figure.

(although it is weakly visible in the presented periodogram for $\mathrm{HF}$ with $N_{\mathrm{f}}=5$, it is already very striking for $N_{\mathrm{f}}=7$, and appears at nearly the same frequency), and one at $f=0.11711 \mathrm{~h}^{-1}$ in data set B. However, they do not appear in the accompanying tests, nor in the original analysis.

Interestingly, the best stability is demonstrated by our shortest-period solutions (i.e. solution F, G, and $\mathrm{H}$ - see Table 2), which are prominent in both test cases. The reason for that is the very simple shape of the corresponding phase profiles the shape which makes them insensitive to small distortions.
In contrast, the solutions at lower frequencies, including those newly found, with more complicated phase profiles, are more sensitive to the searching routine and settings, heliocentric reduction, noise realization and spectral sampling in a specific spectrometer, as well as any geometric and spin instabilities (cf. Appendices C.1-C.3). 


\section{Appendix D: List of the observations}

Table D.1. Observations of the HCN molecule with the AOS spectrometers (see Sect. 2).

\begin{tabular}{|c|c|c|c|c|c|c|c|}
\hline $\begin{array}{c}\text { Date UT } \\
\text { (May 2006) }\end{array}$ & $\underset{\left[\mathrm{K} \mathrm{km} \mathrm{s}^{-1}\right]}{T_{\mathrm{mB}} \mathrm{d} v}$ & $\begin{array}{c}\text { Date UT } \\
\text { (May 2006) }\end{array}$ & $\underset{\left[\mathrm{K} \mathrm{km} \mathrm{s}^{-1}\right]}{T_{\mathrm{mB}} \mathrm{d} v}$ & $\begin{array}{c}\text { Date UT } \\
\text { (May 2006) }\end{array}$ & $\underset{\left[\mathrm{K} \mathrm{km} \mathrm{s}^{-1}\right]}{T_{\mathrm{mB}} \mathrm{d} v}$ & $\begin{array}{c}\text { Date UT } \\
\text { (May 2006) }\end{array}$ & $\underset{\left[\mathrm{K} \mathrm{km} \mathrm{s}^{-1}\right]}{T_{\mathrm{mB}} \mathrm{d} v}$ \\
\hline 10.3167 & $1.29 \pm 0.36$ & 10.6063 & $1.96 \pm 0.28$ & 12.6587 & $1.61 \pm 0.17$ & 20.6469 & $0.26 \pm 0.26$ \\
\hline 10.3201 & $2.08 \pm 0.38$ & 10.6104 & $1.64 \pm 0.33$ & 12.6635 & $1.88 \pm 0.19$ & 20.6517 & $1.11 \pm 0.25$ \\
\hline 10.3243 & $2.43 \pm 0.34$ & 10.6146 & $0.76 \pm 0.32$ & 12.6691 & $1.14 \pm 0.18$ & 20.6573 & $0.61 \pm 0.18$ \\
\hline 10.3278 & $2.01 \pm 0.34$ & 10.6188 & $1.76 \pm 0.35$ & 12.6739 & $1.16 \pm 0.23$ & 20.6622 & $0.94 \pm 0.21$ \\
\hline 10.3320 & $1.94 \pm 0.50$ & 10.6222 & $2.14 \pm 0.43$ & 12.6788 & $1.34 \pm 0.32$ & 20.6670 & $1.05 \pm 0.21$ \\
\hline 10.3361 & $1.25 \pm 0.31$ & 10.6264 & $2.72 \pm 0.35$ & 12.6837 & $1.53 \pm 0.19$ & 20.6719 & $1.42 \pm 0.28$ \\
\hline 10.3396 & $1.36 \pm 0.32$ & 10.6305 & $2.02 \pm 0.49$ & 12.6885 & $1.14 \pm 0.21$ & 20.6767 & $1.03 \pm 0.27$ \\
\hline 10.3438 & $1.68 \pm 0.32$ & 10.6347 & $3.06 \pm 0.32$ & 12.7163 & $1.18 \pm 0.26$ & 20.6823 & $1.51 \pm 0.25$ \\
\hline 10.3472 & $1.95 \pm 0.42$ & 10.6382 & $1.91 \pm 0.43$ & 12.7212 & $1.49 \pm 0.24$ & 20.6872 & $0.64 \pm 0.29$ \\
\hline 10.3514 & $1.53 \pm 0.38$ & 10.6424 & $2.17 \pm 0.49$ & 12.7260 & $1.17 \pm 0.25$ & 20.6920 & $1.12 \pm 0.22$ \\
\hline 10.3555 & $2.31 \pm 0.30$ & 10.6465 & $2.91 \pm 0.40$ & 20.4705 & $2.14 \pm 0.33$ & 20.7260 & $1.06 \pm 0.39$ \\
\hline 10.3590 & $1.42 \pm 0.34$ & 10.6750 & $3.71 \pm 0.65$ & 20.4795 & $1.45 \pm 0.41$ & 20.7309 & $0.73 \pm 0.35$ \\
\hline 10.3632 & $2.16 \pm 0.29$ & 10.6792 & $2.41 \pm 0.55$ & 20.4844 & $1.46 \pm 0.37$ & 20.7358 & $1.48 \pm 0.35$ \\
\hline 10.3674 & $0.82 \pm 0.33$ & 11.6646 & $1.42 \pm 0.16$ & 20.4892 & $1.56 \pm 0.33$ & 20.7406 & $1.75 \pm 0.28$ \\
\hline 10.3715 & $2.40 \pm 0.28$ & 11.6687 & $1.61 \pm 0.17$ & 20.4941 & $0.62 \pm 0.36$ & 20.7455 & $1.71 \pm 0.35$ \\
\hline 10.4361 & $1.61 \pm 0.39$ & 11.6729 & $1.33 \pm 0.18$ & 20.4989 & $2.09 \pm 0.26$ & 20.7497 & $0.98 \pm 0.23$ \\
\hline 10.4403 & $1.88 \pm 0.25$ & 11.6764 & $1.33 \pm 0.23$ & 20.5038 & $1.34 \pm 0.24$ & 20.7545 & $0.76 \pm 0.31$ \\
\hline 10.4445 & $1.71 \pm 0.30$ & 11.6806 & $1.54 \pm 0.17$ & 20.5580 & $1.36 \pm 0.25$ & 20.7594 & $0.94 \pm 0.35$ \\
\hline 10.4486 & $1.03 \pm 0.32$ & 11.6847 & $1.33 \pm 0.17$ & 20.5635 & $1.51 \pm 0.29$ & 20.7691 & $0.55 \pm 0.36$ \\
\hline 10.4535 & $1.37 \pm 0.23$ & 11.6882 & $1.35 \pm 0.19$ & 20.5684 & $1.91 \pm 0.32$ & 20.7739 & $0.82 \pm 0.27$ \\
\hline 10.4576 & $2.15 \pm 0.23$ & 11.6924 & $1.29 \pm 0.18$ & 20.5733 & $1.94 \pm 0.20$ & 22.4642 & $1.19 \pm 0.23$ \\
\hline 10.4618 & $2.01 \pm 0.20$ & 11.6958 & $1.27 \pm 0.18$ & 20.5781 & $1.30 \pm 0.23$ & 22.4684 & $2.35 \pm 0.36$ \\
\hline 10.4667 & $0.98 \pm 0.35$ & 11.7000 & $1.06 \pm 0.21$ & 20.5830 & $1.27 \pm 0.23$ & 22.4733 & $1.06 \pm 0.31$ \\
\hline 10.4708 & $1.08 \pm 0.26$ & 11.7049 & $0.61 \pm 0.18$ & 20.5885 & $0.88 \pm 0.22$ & 22.4781 & $0.62 \pm 0.20$ \\
\hline 10.4757 & $1.82 \pm 0.27$ & 11.7083 & $1.34 \pm 0.23$ & 20.5934 & $1.56 \pm 0.22$ & 22.4830 & $0.59 \pm 0.30$ \\
\hline 10.4805 & $1.45 \pm 0.28$ & 11.7125 & $1.08 \pm 0.19$ & 20.5983 & $0.82 \pm 0.25$ & 22.4879 & $1.02 \pm 0.32$ \\
\hline 10.4854 & $1.08 \pm 0.31$ & 12.3788 & $1.78 \pm 0.21$ & 20.6031 & $1.14 \pm 0.33$ & 22.4927 & $1.02 \pm 0.30$ \\
\hline 10.5903 & $2.12 \pm 0.32$ & 12.3837 & $1.03 \pm 0.26$ & 20.6087 & $1.19 \pm 0.27$ & 22.4976 & $1.59 \pm 0.24$ \\
\hline 10.5945 & $2.37 \pm 0.31$ & 12.6441 & $1.74 \pm 0.18$ & 20.6135 & $1.39 \pm 0.26$ & 22.5024 & $1.38 \pm 0.21$ \\
\hline 10.5986 & $2.51 \pm 0.31$ & 12.6489 & $1.52 \pm 0.21$ & 20.6372 & $1.17 \pm 0.30$ & 22.5073 & $0.58 \pm 0.35$ \\
\hline 10.6021 & $1.80 \pm 0.27$ & 12.6538 & $1.44 \pm 0.21$ & 20.6420 & $1.23 \pm 0.31$ & 22.5122 & $1.65 \pm 0.22$ \\
\hline
\end{tabular}

